\title{
Modelling and Numerical Valuation of Power Derivatives in Energy Markets
}

\author{
Mai Huong Nguyen ${ }^{1, *}$ and Matthias Ehrhardt ${ }^{2}$ \\ ${ }^{1}$ Institut für Mathematik, Technische Universität Berlin, Strasse des 17. Juni 136, \\ 10623 Berlin, Germany \\ ${ }^{2}$ Lehrstuhl für Angewandte Mathematik und Numerische Analysis, Fachbereich \\ C-Mathematik und Naturwissenschaften, Bergische Universität Wuppertal, \\ Gaußstr. 20, 42119 Wuppertal, Germany
}

Received 15 February 2011; Accepted (in revised version) 11 June 2011

Available online 30 April 2012

\begin{abstract}
In this work we investigate the pricing of swing options in a model where the underlying asset follows a jump diffusion process. We focus on the derivation of the partial integro-differential equation (PIDE) which will be applied to swing contracts and construct a novel pay-off function from a tree-based pay-off matrix that can be used as initial condition in the PIDE formulation. For valuing swing type derivatives we develop a theta implicit-explicit finite difference scheme to discretize the PIDE using a Gaussian quadrature method for the integral part. Based on known results for the classical theta-method the existence and uniqueness of solution to the new implicit-explicit finite difference method is proven. Various numerical examples illustrate the usability of the proposed method and allow us to analyse the sensitivity of swing options with respect to model parameters. In particular the effects of number of exercise rights, jump intensities and dividend yields will be investigated in depth.
\end{abstract}

AMS subject classifications: 65M10, 91B25

Key words: Swing options, jump-diffusion process, mean-reverting, Black-Scholes equation, energy market, partial integro-differential equation, theta-method, Implicit-Explicit-Scheme.

\section{Introduction}

In recent years the deregulation process has been developed strongly in the power market in many western countries. Although the establishment of power exchanges was not inevitable, but with its existence, markets gained more transparency and competitiveness. The European Energy Exchange (EEX), Amsterdam Power Exchange

*Corresponding author.

Email: mai.huong@gmx.de (M. H. Nguyen), ehrhardt@math.uni-wuppertal.de (M. Ehrhardt) 
(APX), Nord Pool with Eltermin and Eloption and New York Mercantile Exchange (NYMEX) are the results of the emergence of markets for exchange and trading power.

In most deregulated markets the spot prices do not vary with the season only, one also observes occasional distinctive price spikes which are caused when the maximum supply is close to or lower than the current demand. Such supply congestion occurs when a generator or part of the distribution network fails unexpectedly or sudden change in demand could lead to spikes. Also, transfer of natural gas through pipelines and electricity through transmission lines create additional volume constraints.

The rise of trading electricity delivery contracts aims to satisfy the power requirement on one hand and on the other hand to minimize the new price risks. One of the first types of contracts to be traded on exchange were futures contracts which give the holder the obligation to buy a fixed amount of the commodity for a predefined price at a predetermined time. With many advantages, such as low transaction costs, guaranteed physical delivery and financial performance by the exchange, futures contracts are quite popular in power markets. However, since the owner of a futures contract had to use the ordered amount up or any surplus energy was wasted, this standard contract is not sufficient for market participants who find it difficult to predefine their future need of the commodity, especially when the relevant product cannot be stored or storage is very expensive. Among this immense variety of contracts swing options are the most suitable in hedging extreme price fluctuations of certain commodities. In allowing flexibility-of-delivery with respect to both timing and the amount of energy used, swing contracts can be found in coal markets, gas markets and are especially favored in energy markets, since energy is only storable in a limited manner and its storage process is quite costly e.g., in a pumped-storage power station.

From a mathematical point of view, the pricing of these power derivatives contains several challenges. One is the choice of an appropriate stochastic process which describes the price movement appropriately as electricity spot prices are mean-reverting, exhibit strong seasonalities and jump behaviour. Typically, the spot price, after some sudden jumps, immediately reverts to the long term price level. Another critical aspect is the application of the no-arbitrage approach from financial mathematics presuming markets are liquid meaning that the underlying asset can be bought and sold at a reasonable price at any time. Due to the non-storability and non-trading character of electricity and gas the no-arbitrage approach in financial market can not be applied on power markets without hurdles as buying the underlying today and selling the product in the future is not feasible. However, since the classical arbitrage pricing incorporates many advantages, many authors have been dealing with the question: How can we use arbitrage pricing in incomplete markets?

Instead of modeling the electricity market directly and deriving the forward price, one approach is to model the forward price directly as forward contracts are tradable and hence the no-arbitrage valuation can be applied. Unfortunately, forward contracts are traded only in certain time periods and hence, the liquidity is rather low. For example, the scope of delivery in EEX forward markets starts by a month, a quarter, a year and ends at three years. Thus these forward models do not reflect the price 
behaviour during a day or within an hour which is important for swing options. The other approach is the implicit assumption of liquidly tradable futures contracts for all time points which creates a futures market over the electricity spot market. Therewith the futures prices just in front of delivery present the electricity spot prices and in this way the no-arbitrage pricing methodology is applicable. In line with Wilhelm [22] we choose to use the second approach in the sequel.

In the last ten years research on models with jumps has become very active. In addition to the partial differential term one needs to take account of the integral term when pricing options on an underlying that follows a jump diffusion process. The non-locality of the integro-differential operator is a difficulty when valuating the partial integro-differential equation (PIDE) numerically. To describe the jumps properly one could observe two different approaches, namely solutions were shown for the classical model by Merton [19] and a more recent model by Kou [17]. The former is an attractive model in which the process the asset follows is the sum of a jump process with lognormally distributed jumps and the usual Wiener process. The latter characterizes a pure jump process suggesting a double exponential distribution for the logarithmic jump size. For more details we refer the interested reader to $[17,19]$. In particular, by considering a jump-diffusion model for a single-asset market Almendal and Oosterlee [1] valuated European contingent claim. To tackle the PIDE they used finite differences methods (FDM) and finite elements methods (FEM) to discretize in space and the second order backward differentiation formula (BDF2) in time. Instead of Gaussian quadrature, a fast Fourier transformation (FFT) has been applied for the integral part since the latter is known to be more efficient. Mayo [18] demonstrated a cost reduction of evaluating such integrals by using an operator splitting method to the PIDE and evaluate the integral terms by FFT.

Up to the authors knowledge, only Kjaer [15], Kluge [16] and Kern [14] considered jumps in their swing option valuation but compared to Wilhelm they simplified the model in not allowing the holder to sell energy if they do not belong to the group of producers. According to Kluge [16], Monte-Carlo methods only work for the most basic versions of swing contracts where at each time only one unit of an option can be exercised. Kluge [16] stated that more general swing contracts with a variable volume per exercise and an overall constrained can be priced with a tree based method. Hence, he decided to extend the ideas of Jaillet, Ronn and Tompaidis [13], using a grid rather than a tree allowing spot prices to jump from any value to any other. Kjaer [15] derived a PIDE and based on FDMs and quadrature methods he deduced a numerical scheme to approximate the option value. Among several approaches, FDMs could acquire similar advantages of FEMs and are even easier to implement and thus its popularity has been increased. Wegner [21] presented swing options values as the solution of a partial differential equation (PDE) by extending the classical Black-Scholes equation with stochastic features in the electricity price process, such as seasonality and mean reversion and used FDMs to approximate the solution numerically. Wegner described concisely the pay-off by using lattice methods and even considered penalty features.

In this work we limit ourselves to the classical log-normal, one-factor stochastic 
process for the underlying commodity price to display the price movements. In particular, we do not consider seasonality and mean reversion features as those have been derived and incorporated in the price process by Wegner [21] and Jaillet, Ronn and Tompaidis [13]. We want to focus here on the valuation of power derivatives including jumps. A theoretical foundation for pricing swing options has been derived by Wilhelm [22]. Assuming the existence of a liquid futures market the futures price can be considered as the underlying of swing options. Based on this framework we use the no-arbitrage approach, derive the stochastic differential equation (SDE) for the spot price and develop a PIDE by modifying the Black-Scholes PDE. By extending the ideas of Kjaer [15], who deduced a PIDE for swing options with jumps, we permit any holder to buy as well as to sell the underlying. A numerical scheme based on FDMs and quadrature routines is derived which offers high flexibility regarding spot price models and pay-off functions. To justify the complex features of swing options, we will generate a pay-off function from a tree-based pay-off matrix, hereby extending the ideas of Wegner [22]. After describing the numerical scheme for swing options and presenting numerical examples, we will investigate and interpret the dependence of swing option values on typical process parameters like number of exercise rights, jump intensities and dividend yields.

The paper is organized as follows. In Section 2 we describe swing options including a discussion of boundary conditions, special cases and the pay-off function. We will propose and examine in detail a simple log-normal, one-factor model exhibiting price spikes for the spot price of the underlying commodity and deals with the derivation of the PIDE for swing options. Section 3 focuses on valuation of swing options based on FDMs with Gaussian quadrature methods for the integral part. A proof of the stability and convergence of the numerical scheme is provided. Finally, in Section 4 swing option's pricing results in dependence of model parameters are presented.

\section{Swing options}

Swing contracts are a vast species of options and traded generally as over-the-counter (OTC) contracts, meaning traders are private parties. The option's purchaser often combines swing options with a set of forward contracts. Latter oblige the holder to buy or sell a certain amount $b_{i}$ of the underlying for a set of predefined strike prices $K_{i}$ at a discrete set of dates $\left\{t_{1}, \cdots, t_{n}\right\}$ over a certain period $t_{i} \in\left[t_{0}, T\right], \forall i \in\{1, \cdots, n\}$.

With the swing contract the holder has the rights to sell or buy the underlying on top of the volume $b_{i}$ specified in forward contracts multiple times during a predetermined contract period $\left\{t_{1}, \cdots, t_{n}\right\} \subseteq\left[t_{0}, T\right]$ at a set of strike prices $K_{j}, j \in\{1, \cdots, N\}$. However, at a time $t_{j}, j \in\{1, \cdots, N\}$, the holder is restricted to exercise only one right, whereas $t_{j}$ typically denotes a day or a week. To make it short, the swing option holder has $n$ opportunities to exercise his $N$ rights. It does not make sense to have more rights than opportunities since you can not exercise more than one right at one opportunity. Thus, we postulate the condition $N \leq n$. 
If the exercise time is continuous, that means the holder may buy or sell at any time until maturity $t_{j} \in\left[t_{0}, T\right]$, swing derivatives can be viewed as a portfolio of American options. However, due to the restriction of delivery process there is always a minimum distance of two exercise dates which is called the refraction time. If the exercise time is discrete, that means the holder has the right to purchase or sell at a predefined discrete set of dates $\left\{t_{1}, \cdots, t_{n}\right\}$, swing derivatives can be seen as a portfolio of either Bermudan options or European options. Note that it makes sense to set the last exercise opportunity $t_{N}$ equal to $T$.

In most cases not only the total number of rights is restricted by $N$, but also a volume restriction is given. There are lower and upper bounds for each right, such as

$$
\Delta_{j} \in\left(l_{1}^{j}, l_{2}^{j}\right) \cup\left(l_{3}^{j}, l_{4}^{j}\right) \quad \text { with } l_{1}^{j} \leq l_{2}^{j} \leq 0 \leq l_{3}^{j} \leq l_{4}^{j}, \forall j \in\{1, \cdots, N\} .
$$

If $\Delta_{j}$ is negative, the holder is allowed to consume $\Delta_{j}$ less while the predetermined amount $b_{j}$ must be purchased. So what the investor can do is to sell exactly this amount $\Delta_{j}$ from $b_{j}$ to a third party and hence the consumption in $t_{j}$ with $b_{j}+\Delta_{j}$ reflects a swing put option or a downswing, see [7]. If $\Delta_{j}$ is positive, in addition to $b_{j}$, the holder has the right to buy $\Delta_{j}$ and create therewith a call or a upswing, respectively. Hence, a swing option is characterized by one number $\Delta_{j}$. The total number of downswings $d$ and upswings $u$ could be restricted with $d \leq D$ and $u \leq U$.

Besides, an overall consumption constraint is common, e.g.,

$$
C=\sum_{j=1}^{N} b_{j}+\Delta_{j} \leq \text { Max. }
$$

The so-called penalty function $\rho$ is related to this limitation (2.2) allowing the holder to exceed these limits, but in connection with a monetary fine. Let $x$ denote the total consumption in $\left[t_{0}, T\right]$ and $\mathbf{E}\left[S_{t_{0}} \mid S_{T}\right]$ the expectation of the commodity's spot price at the end of the contract. Then the penalty function in $x$ is defined by

$$
\rho(x)= \begin{cases}C, & \text { if } x<\operatorname{Min}, \\ 0, & \text { if } \operatorname{Min} \leq x \leq \operatorname{Max}, \\ (x-\operatorname{Max}) \mathbf{E}\left[S_{t_{0}} \mid S_{T}\right], & \text { if } x>\operatorname{Max},\end{cases}
$$

with given parameters Min, Max $\in \mathbb{R}$. Therefore swing options are also called takeor-pay options. To simplify the matter we assume that the strike prices $K_{j} \equiv K, \forall j \in$ $\{1, \cdots, N\}$ are identical. As we are only concerned with the time to expiry of the contract, we can assume, without loss of generality, that the present time $t_{0}$ is zero and the interval $[0,1]$ denotes a period of one year.

In this paper we concentrate on valuating swing options of Bermudan type. The boundary conditions for a Bermudan option are the same as for an American option if $t \in\left[t_{1}, \cdots, t_{n}\right]$ for $t_{i}$ denotes one exercise opportunity $\forall i \in\{1, \cdots, n\}$. Otherwise the boundary conditions for a Bermudan option are the same as for a European option. 


\subsection{Special cases}

Although swing options are many fold we can still deduce some conclusions based on the characteristics of exercise rights and opportunities. There are some articles on this specification, see e.g., $[7,13,21]$, but most of them only deal with one type of exercise times, namely a set of discrete dates. According to [13,21], the value of a swing option with one right is equal to the value of a Bermudan option, but this does not hold in general as the option value depends not only on the number of rights but also on the type of exercise time, whether it is a fixed date, a set of dates or a period of time. For instance, Wilhem [22] stated that swing options with one right is equal to the value of an American option since she considered the exercise time as any element of a period of time interval. Presuming there is no penalty for swing options we could state in the sequel some important cases.

\subsubsection{Continuous exercise time}

The exercise time $t_{j}$ is continuous if $t_{j} \in[0, T], \forall j \in\{1, \cdots, N\}$, where $N \in \mathbb{N}$ denotes the number of exercise rights.

- If $N=1$, the option holder has one right and may exercise any time until maturity, i.e., the value of a swing option is equal to the value of an American option.

- If $N>1$, then the value of a swing option is never larger than the value of a portfolio of American options.

\subsubsection{Discrete exercise time}

The exercise time $t_{j}$ is discrete if $t_{j} \in\left\{t_{1}, \cdots, t_{n}\right\} \subseteq[0, T], \forall j \in\{1, \cdots, N\}$, where $N \in$ $\mathbb{N}$ denote the number of exercise rights and $n \in \mathbb{N}$ number of exercise opportunities.

- If $N=1$ and $n=1$, then at a prespecified time $t_{1}$ the option holder has the right to exercise once. Thus, the value of a swing option is equal to the value of a European option.

- If $N=1$ and $n>1$, then at a predefined set of time the option holder has the right to exercise once. Thus, the value of a swing option is equal to the value of a Bermudan option.

- If $N=n>1$, then the number of rights is equal to the number of dates. Thus, the value of a swing option is equal to the value of a portfolio of European options.

- If $1<N<n$ and as long as $N<n$, we have more exercise opportunities for one right. But as soon as $N=n$, we arrive at the case mentioned above. Thus, the value of a swing option is never larger than the value of a portfolio of Bermudan options and European options.

\subsubsection{Conclusions for upper and lower bounds}

With these results we derive bounds for swing options which are extremely helpful for a better understanding and verifying the calculated swing option prices. 


\section{Continuous exercise time}

- Upper bound: The value of swing options is equal to the value of a bundle of American options only when the holder of such options never exercises more than one option at the same time. In other words, the value of a portfolio of American options is greater than the value of a swing option as soon as the holder of the first portfolio exercises at least two American options together. Thus the upper bound is given by the sum of American options.

\section{Discrete exercise time}

- Upper bound: Compared to a swing option's holder, who only has one exercise right at a predefined time, a Bermudan option's holder has the advantage to exercise all at once. Therefore the upper bound is the sum of Bermudan options.

- Lower bound: As a Bermudan's option value is never smaller than a European one, the lower bound is hence given by the sum of European options.

For the sake of completeness we have presented upper and lower bounds for both discrete and continuous exercise times, but in the sequel we will focus on the discrete case and on swing options of Bermudan types.

\subsection{A partial integro-differential equation for swing option pricing}

In this section we construct a non-linear PIDE which describes swing options very well. The standard assumptions for Black-Scholes PDE are: no arbitrage, no dividend and transaction costs, constant interest rate $r$ and volatility $\sigma$ and the asset price $S$ satisfies the following stochastic differential equation

$$
\frac{\mathrm{d} S}{S}=\mu \mathrm{d} t+\sigma \mathrm{d} X
$$

In order to adapt the original Black-Scholes framework to swing options we need to modify a few assumptions and therefore extend the Black-Scholes PDE

$$
\frac{\partial V}{\partial t}+r S \frac{\partial V}{\partial S}+\frac{\sigma^{2} S^{2}}{2} \frac{\partial^{2} V}{\partial S^{2}}=r V
$$

to a PIDE. It is a well-known fact that Bermudan options are discretized American options and hence for swing options we have to dismiss the no dividend assumption. Here we only consider deterministic dividends whose value is fixed at the beginning of the option's contract. This assumption is not too restrictive since dividend payments in many companies stay similar within many years. The dividend payment of an asset at a certain time $\mathrm{d} t$ is denoted as $\delta S \mathrm{~d} t$. As the dividend yield is defined as the ratio of the dividend payment to the asset price, the dividend $\delta S \mathrm{~d} t$ therefore represents a constant and continuous dividend yield. Hence, whenever the underlying 
asset pays out dividends, the asset price must fall by the same amount as otherwise arbitrage opportunities would exist. The asset price $S$ is therefore modified to

$$
\mathrm{d} S=(\mu-\delta) S \mathrm{~d} t+\sigma S \mathrm{~d} X,
$$

and the corresponding PDE then reads

$$
\frac{\partial V}{\partial t}+\frac{\sigma^{2} S^{2}}{2} \frac{\partial^{2} V}{\partial S^{2}}+(r-\delta) S \frac{\partial V}{\partial S}=r V
$$

The stock price does not have only continuous changes, but also jumps. One can observe this behaviour in energy prices and in most cases swing options are energy derivatives. That is why a constant volatility $\sigma$ is not realistic at all. For the purpose of evaluating options with jumps there exist two popular jump-diffusion models. The classical Merton's model uses a log-normal distribution for the log jump size whereas the more recent Kou's model assumes the price of the underlying asset behaves like a geometrical Brownian motion (GBM) with a drift and jumps whose size is log-double-exponentially distributed. Both are attractive models since analytical formulae for pricing vanilla options with jumps exist, and with the latter model some path-dependent options such as lookbacks, barriers and perpetual Americans with jumps are tractable analytically. For more details of Merton's and Kou's model we refer to $[17,19]$. Recently Almendral and Oosterlee [1] considered both models for a single-asset market. After deriving a PIDE for a European option with jumps they compared the numerical solutions to the analytical solutions. Similar studies were done by Mayo [18] and Toivanen [20], where in addition to European options American options are also considered. For the consideration of jumps we choose the Merton model as this model yields the same results and is less complicated to handle. For modelling spikes Merton suggested the Poisson process which is presumed to be independent of the Brownian part. According to [3] the modified one-factor SDE becomes

$$
\mathrm{d} S=(\mu-\delta) S \mathrm{~d} t+\sigma S \mathrm{~d} X+(q-1) S \mathrm{~d} Q,
$$

where $\mathrm{d} Q$ indicates a Poisson process of intensity $\lambda$ with

$$
P[\mathrm{~d} Q=0]=1-\lambda \mathrm{d} t, \quad P[\mathrm{~d} Q=1]=\lambda \mathrm{d} t,
$$

and $q \in[0, \infty]$ is a random variable which is log-normally distributed with the density

$$
\Gamma_{\gamma, \mu_{j}}(q)=\frac{1}{\sqrt{2 \pi} \gamma} \exp \left(-\frac{1}{2}\left(\frac{\log (q)-\mu_{j}}{\gamma}\right)^{2}\right),
$$

with the obvious properties of being non-negative and

$$
\int_{0}^{\infty} \Gamma_{\gamma}(q) \mathrm{d} q=1, \quad \forall q \in[0, \infty] .
$$

Note that $q-1$ is called the impulse function, which produces a jump from $S$ to $S q$ and $\mathbb{E}(q-1)=\bar{k}$ denotes the expected relative jump size, cf. [4]. If $0 \leq q<1$, the negative 
impulse function causes the spot price to decrease. If $q=1$, the spot price remains the same. If $q>1$, the positive impulse function causes the spot price to increase.

Based on the Feynman-Kac formula, which states the equivalence between expectation values and PIDEs, we can derive a PIDE that governs swing options whose underlying's spot price $S$ follows the process (2.8), namely

$$
\frac{\partial V}{\partial t}+\frac{\sigma^{2} S^{2}}{2} \frac{\partial^{2} V}{\partial S^{2}}+(r-\delta-\lambda \bar{k}) S \frac{\partial V}{\partial S}-r V+\lambda \int_{0}^{\infty}(V(q S, t)-V(S, t)) \Gamma_{\gamma}(q) \mathrm{d} q=0 .
$$

Generalization to the case of multiple assets can be found in [16] or [14]. For further understanding how to deduce a PIDE we refer to Kluge [16], where the Feynman-Kac formula has been used to derive a PIDE for swing options from a two-factor model SDE. However, there have not been any attempt to analyse this PIDE numerically. That is why we limit ourselves to modelling a swing option on one given underlying asset as we want to focus on the numerical valuation of this PIDE.

Intuitively, one can explain (2.8) this way: the spot price follows randomly a GBM stochastic process [2]. Whenever having a sudden jump the price will return to the GBM trend. A positive jump means a significant higher price than usual and a negative jump means a lower price than average. Consequently, the curve describing the spot price $S$ with respect to time $t$ will be continuous differentiable most of the time apart from finite negative or positive jumps. In order to perform the discretization some transformations of variables are necessary. By the substitution of $t=T-\tau$, the time to maturity, we manage to reverse the time direction and therewith to turn the resulting equation into a forward-in-time equation. The expiry $T$ now matches to $\tau=0$ and the issue time $t=0$ matches to $\tau=T$. The domain of the values $S$ of the underlying is bounded from below by zero: $0 \leq S \leq \infty$. When transformed by $x=\ln (S)$ the spot price corresponds to whole real line: $-\infty<x<\infty$. By these standard transformations, we intend to get all the coefficients constant which is necessary for our next steps. In summary, we obtain

$$
\begin{array}{ll}
\tilde{V}(x, \tau)=V\left(e^{x}, T-\tau\right)=V(S, t), & \\
\frac{\partial \tilde{V}}{\partial \tau}=-\frac{\partial V}{\partial t}, \quad \frac{\partial V}{\partial S}=\frac{1}{S} \frac{\partial \tilde{V}}{\partial x}, \quad \frac{\partial^{2} V}{\partial S^{2}}=\frac{1}{S^{2}} \frac{\partial^{2} \tilde{V}}{\partial x^{2}} \\
q \in[0, \infty], \quad S \geq 0 \Rightarrow S q \in[0, \infty], \quad \ln (q), x \in \mathbb{R} .
\end{array}
$$

The PIDE therefore reads

$$
\frac{\partial \tilde{V}}{\partial \tau}=\frac{\sigma^{2}}{2} \frac{\partial^{2} \tilde{V}}{\partial x^{2}}+(r-\delta-\lambda \bar{k}) \frac{\partial \tilde{V}}{\partial x}-r \tilde{V}+\lambda\left(\int_{-\infty}^{\infty} \tilde{V}(z+x, \tau) \Gamma_{\gamma}(z) \mathrm{d} z-\tilde{V}\right),
$$

with the corresponding pay-off function $\tilde{V}(x, 0)$ which is Lipschitz continuous.

Following [4], Eq. (2.11) can be seen as a particular case of the linear parabolic integro-differential equation in $\mathbb{R} \times(0, T)$

$$
\tilde{V}_{\tau}=a \tilde{V}_{x}+b \tilde{V}_{x x}+c \tilde{V}+\lambda\left(\int_{-\infty}^{\infty} \tilde{V}(z+x, \tau) \Gamma_{\gamma}(z) \mathrm{d} z-\tilde{V}\right),
$$


where $a=(r-\delta-\lambda \bar{k}), b=\sigma^{2} / 2$ and $c=-r$ are all constants.

As $q$ is log-normally distributed, $\mathbb{E}(q)=\exp \left(\mu_{j}+\gamma^{2} / 2\right)$ and therefore the expected relative change in the underlying price is given by $\bar{k}=\exp \left(\mu_{j}+\gamma^{2} / 2\right)-1$, $z=\ln (q) \in \mathbb{R}$ is normally distributed with the density function

$$
\Gamma_{\gamma, \mu_{j}}(z)=\frac{1}{\sqrt{2 \pi} \gamma} \exp \left(-\frac{1}{2}\left(\frac{z-\mu_{j}}{\gamma}\right)^{2}\right)
$$

\subsection{Initial and boundary conditions}

Having derived the PIDE for the value of the swing options, we should next impose initial conditions and boundary conditions.

To derive the initial conditions means to deduce the pay-off function for swing options, which is more difficult than dealing with single-right options, such as European or American options. The pay-off function models the outcome at the end of the contract which is the last exercise opportunity in our case. As swing options depend on many factors, we will first list the relevant parameters.

- Number of allowed upswings $U$ and Number of allowed downswings $D$.

- Number of exercised upswings $u$ and Number of exercised downswings $d$.

- Last exercise opportunity $t_{n}, n$ denotes the number of exercise opportunities.

- A set of strike prices $K_{j}$ (to simplify the problem we set $K_{j} \equiv K, \forall j$ ).

- Amount of consumption per exercise right $\Delta_{j}$ with the presumption $\Delta_{j}$ being constant for all exercise opportunities, i.e., $\Delta_{j} \equiv \Delta$. If the consumption is negative, we denote $\Delta_{\downarrow} \leq 0$, otherwise, $\Delta_{\uparrow} \geq 0$.

- The penalty function $\rho$, where $\rho^{u, d}=\rho\left(\Delta_{\downarrow} u+\Delta_{\uparrow} d\right)$ denotes the cost for exceeding the overall consumption Max with the property $\rho^{0,0}=\rho^{n, n}, \forall n \in \mathbb{N}$.

There are many references concerning pay-off functions of swing options, e.g., [7,16, $21,22]$, but most of them describe the pay-off function stochastically [16,22].

To the authors' knowledge, Wegner [21] was the first to use lattice methods to deduce the pay-off function for swing options. The main advantage of this approach is the less complicated embedding of the different constellations of upswings, downswings and exercise opportunities in the pay-off function. The disadvantage of this methods is that the exercise time must be discrete and thus swing options of American type cannot be considered in the pay-off function. However, there are still ways to handle this problem. To approximate swing options of American type with swing options of Bermudan type the set of dates $\left\{t_{1}, \cdots, t_{n}\right\}$ can be made as large as possible. Also, one can impose a constraint on the swing option's value of American type, namely the swing option's value should never be less than its pay-off as otherwise arbitrage opportunities would exist.

Dörr [7] made a further investigation on constructing pay-off functions of swing options for different exercise strategies. He noticed that "the benefit from immediate 
exercise is not only the pay-off, but the pay-off plus the value of the remaining swing option". In other words, the pay-off of swing options is not only the pay-off from the last opportunity but the pay-off plus all previous pay-offs. Dörr [7] also realized that, because of penalty terms of the earlier exercised opportunities, the pay-off functions of [21] might have negative values which can be seen in [21, Eq. (4.14)]. For example, if $K_{\downarrow v}-S_{j}<0$, then the initial value in this case is exactly the penalty for exceeding the given consumption volume. Therefore, Dörr's [7] initial condition is Wegner's [21] initial condition but without penalty terms of the previous pay-off. Only the pay-off on the last exercise opportunity will be treated in his initial conditions. Based on the Least-Squares-Monte-Carlo approach, he sets up a cash flow matrix and incorporated all possible pay-offs of earlier exercise opportunities in this matrix.

In line with [21], we have chosen to evaluate swing options by formulating the problem as a PIDE and thus, we cannot use the approach of Dörr [7]. Hence, in the following we will rewrite Wegner's initial conditions [21] so that non-negativity is guaranteed. Arriving at the last exercise opportunity the option holder could whether have already used all upswing rights or all downswing rights or none of them. The penalty function comes into the picture only if one exceeds the overall constraint. Therefore, we need to consider a penalty at the last exercise opportunity.

When calculating the pay-off of swing options we are not only interested in the pay-off at the last opportunity but also in the pay-off at all others opportunities. Since we do not consider the main characteristics of swing options in our PIDE, the multiple early exercise features necessarily have to show up in the initial condition. Otherwise the pay-off of swing options is equal to the pay-off of a European option, which is a quite unrealistic assumption, especially when one considers a penalty for the early exercising like Wegner [21]. Our extended description of the pay-off functions is:

$V^{u, d}$ : Having exercised $u$ upswings and $d$ downswing the holder has the choice to keep the remaining rights and gets the pay-off of the already exercised rights minus possible penalty, to buy some or to sell some to get more profit minus possible penalty. If $u=U$ and all upswings are used, then the possibility of buying is zero. The same holds for downswings. Note that in contrast with Wegner [21] and Dörr [7] we consider the pay-off of already exercised rights and ensure the non-negativity of the pay-off function.

Assuming there are two strike prices whereas $K_{c}$ denotes the strike price for the upswings and $K_{p}$ denotes the strike price for the downswings, by using the change of variables $x=\ln (S)$ and the time reversal $t=T-\tau$ the description reads

$$
\begin{aligned}
\tilde{V}^{u, d}(x, 0)=\max \{ & \max \left\{u \Delta_{\uparrow}\left(e^{x}-K_{c}\right), d \Delta_{\downarrow}\left(K_{p}-e^{x}\right)\right\}-p^{u, d}, \\
& \max \left\{(u+1) \Delta_{\uparrow}\left(e^{x}-K_{c}\right), d \Delta_{\downarrow}\left(K_{p}-e^{x}\right)\right\}-p^{u+1, d}, \\
& \left.\max \left\{u \Delta_{\uparrow}\left(e^{x}-K_{c}\right),(d+1) \Delta_{\downarrow}\left(K_{p}-e^{x}\right)\right\}-p^{u, d+1}, 0\right\}, \\
\tilde{V}^{u, d}(x, 0)=\max \{ & \max \left\{U \Delta_{\uparrow}\left(e^{x}-K_{c}\right), d \Delta_{\downarrow}\left(K_{p}-e^{x}\right)\right\}-p^{u, d}, \\
& \left.\max \left\{U \Delta_{\uparrow}\left(e^{x}-K_{c}\right),(d+1) \Delta_{\downarrow}\left(K_{p}-e^{x}\right)\right\}-p^{u, d+1}, 0\right\},
\end{aligned}
$$




$$
\begin{gathered}
\tilde{V}^{u, D}(x, 0)=\max \left\{\max \left\{u \Delta_{\uparrow}\left(e^{x}-K_{c}\right), D \Delta_{\downarrow}\left(K_{p}-e^{x}\right)\right\}-p^{u, D},\right. \\
\left.\max \left\{(u+1) \Delta_{\uparrow}\left(e^{x}-K_{c}\right), D \Delta_{\downarrow}\left(K_{p}-e^{x}\right)\right\}-p^{u+1, D}, 0\right\}, \\
\tilde{V}^{U, D}(x, 0)=\max \left\{\max \left\{U \Delta_{\uparrow}\left(e^{x}-K_{c}\right), D \Delta_{\downarrow}\left(K_{p}-e^{x}\right), 0\right\}-p^{U, D}, 0\right\} .
\end{gathered}
$$

Based on these equations above we have to compute a pay-off matrix of size $V=$ $[U+1, D+1]$ in the worst case. Depending on the relationship of $U, D$ and $N$, the size of our pay-off matrix will be different.

In the following a description of this relationship will be presented in detail for three cases for all $U, D$ and $N \in \mathbb{N}$ with $N \geq 2$. For $N=0$, we have $V=[0,0]$ and for $N=1$, we have $V=[1,1]$. Given the number of upswings $U$, downswings $D$ and exercise opportunities $N$ we can detect the size of the pay-off matrix as follows

- First Case: $U+D=N$

(a) For $U=0$ and $D \neq 0 \Rightarrow V=[1, D]$.

(b) For $U \neq 0$ and $D=0 \Rightarrow V=[U, 1]$.

(c) For $U=n, D=m \Rightarrow V=[U+1, D+1], \forall n, m \in \mathbb{N}^{+}$.

Note that the field $V(D+1, U+1)$ does not possess a value!

- Second Case: $U+D<N$

(a) For $U=n, D=m \Rightarrow V=[U+1, D+1], \forall n, m \in \mathbb{N}^{+}$.

It is worth noting that it does not matter how large the opportunities and rights are, the pay-off is always the same, which is unrealistic. Hence we want to introduce an artificial value $\log (N+n)$, which will be multiplied to the last field $V(D+1, U+1)$. Doing so we can achieve the following strong monotony of $V$ :

$$
\begin{array}{lll}
V(u, d, N+1)>V(u, d, N), & \forall(u, d) \in[U, D], \quad N \in \mathbb{N}, \\
V(u, d, n+1)>V(u, d, n), & \forall(u, d) \in[U, D], \quad n \in \mathbb{N} .
\end{array}
$$

- Third Case: $U+D>N$

(a) For $U=n, D=m, \forall n, m \in \mathbb{N}$

- If $U \leq D$ and $U<N \Rightarrow V=[U+1, N]$.

- If $D \leq U$ and $D<N \Rightarrow V=[N, D+1]$.

- If $N \leq D$ and $N \leq U \Rightarrow V=[N+1, N+1]$.

Suppose an option holder has three rights in total. Based on Wegner's description [21] the corresponding pay-off lattice (Fig. 1) leads to the following pay-off matrices

$$
P_{1}:=\left(\begin{array}{cccc}
V^{3,0} & 0 & 0 & 0 \\
V^{2,0} & V^{2,1} & 0 & 0 \\
V^{1,0} & V^{1,1} & V^{1,2} & 0 \\
0 & V^{0,1} & V^{0,1} & V^{0,3}
\end{array}\right), \quad P_{2}:=\left(\begin{array}{ccc}
V^{2,0} & V^{2,1} & 0 \\
V^{1,0} & V^{1,1} & V^{1,2} \\
0 & V^{0,1} & V^{0,1}
\end{array}\right) .
$$




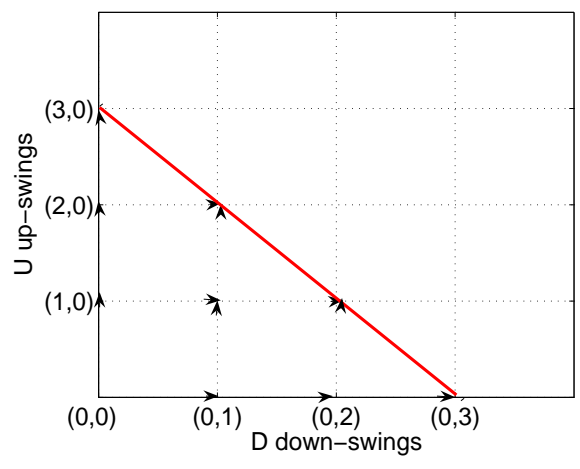

(a) Number of upswings and downswings allowed are each nominated with 3 .

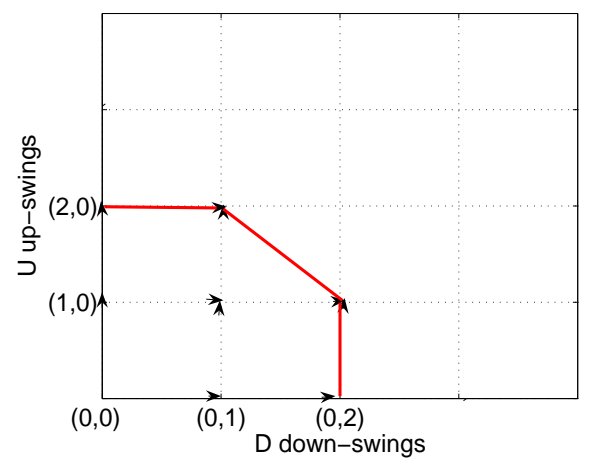

(b) Number of upswings and downswings allowed are each nominated with 2.

Figure 1: The arrows at the lattice points show possible decisions of the swing option's holder. Arriving at the last opportunity the owner could have used up all rights or none. The diagonal line represents the limit $U+D \leq N$.

It is important to note that the pay-off of the triple $(U, D, N)=(1,1,1)$ is equal to the triple $(1,1,2)$ which is one of a few exceptions based on the assumption about the spot price's movement. The possibility that the spot price of the underlying jumps from 0 to $2 \mathrm{~K}$ within two arbitrary exercise opportunities is almost zero.

Having calculated the pay-off functions and saved them in one pay-off matrix, there exist two ways to compute one pay-off function. Either we impose the probability of every node, then the probability of the holder exercising an upswing is defined as $\mathfrak{u}$ and downswing as $\mathfrak{d}$, respectively. The probability of holding the rights is then $1-\mathfrak{u}-\mathfrak{d}$. Hence, the probability of arriving at the last opportunity without exercising any right is $(1-\mathfrak{u}-\mathfrak{d})^{N-1}$, where $N$ denotes the number of exercise rights. However, the probability of buying, selling and staying put would not remain the same if the number of allowed up or down swings are used up but exercise opportunities are still available. Besides, we have to determine the probability which is not reasonable. Therefore, we choose not to follow this idea, but to compute the average of all relevant fields in the pay-off matrix.

Algorithm 2.1. For each node $(i, j)$ a pay-off function is calculated which will be saved as a vector since we have to compute the pay-off for all spot prices. The quotient of numb, the sum of all pay-off functions, yields the desired initial condition.

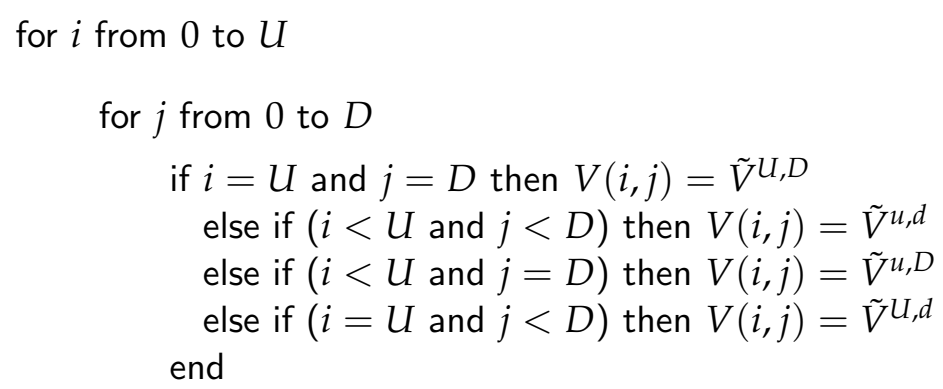




$$
V=\operatorname{sum}(V(0: U, 0: D)) /(\text { numb })
$$

Example 2.1. Suppose a swing option holder has 12 rights to exercise at 50 opportunities at a strike price of $15 €$ per MWh.

In Fig. 2 we present several pay-off functions for this swing option with different numbers of upswings and downswings allowed.

The effect of different exercise rights is illustrated in Fig. 2, where the holder with the highest pay-off is also the holder possessing the most number of upswings and downswings, namely $(U, D)=(12,12)$. Even the number of exercise opportunities and exercise rights remain constant at 50 and 12, the values of the pay-off functions decreased by decreasing number of upswings and downswings allowed.

The specification of proper boundary conditions for swing options is necessary for the numerical scheme. The boundary conditions with respect to $x$ prescribe the values for $\tilde{V}$ in the boundaries of the domain of $\tilde{V}$. Since $S \in[0, \infty)$ and thus $x=\ln (S) \in \mathbb{R}$, the boundary conditions must describe the asymptotic behaviour for $x \rightarrow \pm \infty$.

Comparing to initial conditions there are only a few literatures about boundary conditions for swing options. Kluge [16] wrote a small part about boundary conditions, namely about the value of swing options with no exercise rights being zero for all spot prices and to any time. Dörr [7], on the other hand, treated boundary conditions only in the context of upper and lower bounds. In line with [21], we have found out that there is no evidence about the behaviour of swing options as a linear function when the asset price becomes very small or very large. Due to the possibility of containing both call and put features it is quite clear that swing options could be polynomials of second degree. This remarks our first obstacle since monotony of the relevant derivatives in the spot price is an important property to simplify the investigation about boundary conditions for derivative valuation [6]. One way to tackle this issue is to follow [21] and use one-sided derivatives for the spatial discretization at the boundaries instead of imposing boundary conditions. The disadvantage of this approach is the reduced order of convergence.

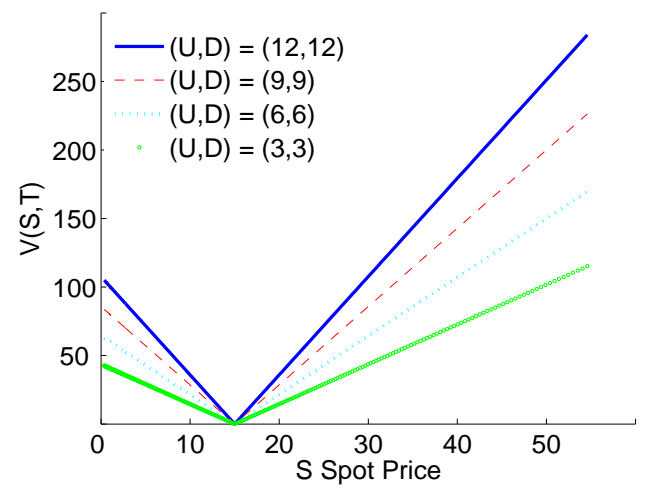

Figure 2: Pay-off functions of a swing option on Example 2.1. No penalty is considered. 
Wilhelm [22] investigated boundary conditions for swing options in more detail. On the basis of the excess to pay-off functions, which model the distance between the option value and their pay-off, and knowing the fact that the localization error for the excess to pay-off function decays exponentially with $N \rightarrow \infty$, Wilhelm [22] first imposes homogeneous Dirichlet boundary conditions for European and American options and in analogy to this she chooses the same approach to determine the boundary for swing options. Wilhelm [22] computed the pay-off function iteratively and so its exact values are not known in advance. Thus she replaces the pay-off function by an artificial deterministic function with the same asymptotic behaviour. We already have a deterministic pay-off function. Hence we define the excess to the pay-off function as

$$
\hat{V}^{u, d}(\tau, x):=\tilde{V}^{u, d}(\tau, x)-\tilde{V}^{u, d}(0, x), \forall(\tau, x) \in[0, T] \times \mathbb{R}, \forall(u, d) \in[0, U] \times[0, D] .
$$

If the swing options had a characteristic of European options with a constant interest rate $r$, then the excess to pay-off function reads

$$
\hat{V}^{u, d}(\tau, x):=\tilde{V}^{u, d}(\tau, x)-e^{-r} \tilde{V}^{u, d}(0, x), \forall(\tau, x) \in[0, T] \times \mathbb{R}, \forall(u, d) \in[0, U] \times[0, D] .
$$

Thus, due to the exponential decay of the excess to pay-off function we could impose the homogeneous Dirichlet boundary conditions on $\hat{V}$ and therewith determine the boundary conditions for swing options:

$$
\hat{V}^{u, d}(\tau, x) \geq 0, \forall(\tau, x) \in[0, T] \times[-M h, N h], \forall(u, d) \in[0, U] \times[0, D] .
$$

\section{Special Cases}

The boundary conditions for European options are well-known. For a call we have

$$
\lim _{S \rightarrow 0} C(S, t)=0, \quad \lim _{S \rightarrow \infty} C(S, t)=S e^{-\delta(T-t)}-K e^{-r(T-t)} .
$$

And for a put we have the boundary conditions

$$
\lim _{S \rightarrow 0} P(S, t)=K e^{-r(T-t)}, \quad \lim _{S \rightarrow \infty} P(S, t)=0,
$$

where $K$ denotes the strike price and $T$ the expiry date. So if the number of rights $N$ is equal to the number of exercise opportunities $n$ then we can deduce boundary conditions for swing options, where $U$ and $D$ denote the number of up and down swings allowed. We can summarize them in two equations, namely

$$
\lim _{x \rightarrow-\infty} \tilde{V}^{U, D}(x, \tau)=D K e^{-r \tau}, \quad \lim _{x \rightarrow \infty} \tilde{V}^{U, D}(x, \tau)=U\left(e^{x-\delta \tau}-K e^{-r \tau}\right) .
$$

And again, for different strike prices $K_{p} \neq K_{c}$ the boundary conditions read

$$
\lim _{x \rightarrow-\infty} \tilde{V}^{U, D}(x, \tau)=D K_{p} e^{-r \tau}, \quad \lim _{x \rightarrow \infty} \tilde{V}^{U, D}(x, \tau)=U\left(e^{x-\delta \tau}-K_{c} e^{-r \tau}\right) .
$$

With these results we can calculate swing options of European type which is indeed a helpful result to verify numerical solutions, since we have derived that a portfolio of $n$ European options show a lower boundary for a swing option with the same rights and opportunities. To check the upper boundary we need the boundary conditions for swing options possessing higher exercise opportunities than exercise rights. 


\section{Numerical analysis}

Even without the integral part in our derived PDE, the convection-diffusion part of the PIDE, does not have an exact or closed solution due to its early exercise feature. In the last few years, there have been several suggestions how to solve these problems. Toivanen [20] studied the pricing of European and American options under Kou's model and his main contribution was the treatment of the integral term. Due to the log-double-exponential form of the kernel in Kou's model, he successfully derived recursion formulae for evaluating the integrals. Mayo [18] also priced European and American options using both Kou's and Merton's methods. She presented a rapid method for evaluating the integrals in the Merton model using the fact that at any time the correlation integral is a translated solution of the heat equation. By applying the FFT on the correlation integral she reduced the computational cost enormously.

Kjaer [15] and Kluge [16] derived a PIDE for pricing swing options with jumps, but they simplified the matter in not allowing the holder to sell energy if they do not belong to the group of producers. Kjaer [15] carried out a numerical analysis for the PIDE. By using the operator splitting method the diffusion part was discretized by the Crank-Nicolson method and the integral part by an explicit scheme.

For the integral problem let us refer to Duffy [8] who showed how to get the finite integral in order to use the Gaussian quadrature. The special property of the integral in Merton-type models is that the kernel of the integral decays rapidly. Although there is a numerical approximation for infinite integrals, but based on this advantage it is much more convenient and requires less effort to choose the integral boundary $A$ and $B$ such that the difference between the original term and the term with the new boundary is not bigger than a given constant

$$
\left|\lambda\left(\int_{-\infty}^{\infty}-\int_{A}^{B}(\tilde{V}(z+x, \tau)-\tilde{V}(x, \tau)) \Gamma_{\gamma, \mu_{j}}(z) \mathrm{d} z\right)\right| \stackrel{!}{<} \tilde{\epsilon} .
$$

Let us have a closer look at the integrand which consists of two functions. One is the unknown solution and the other is the probability density function $\Gamma_{\gamma}(z)$ which tends to zero for $z \rightarrow \pm \infty$. Hence, we are only interested in the finite domain in which the density function is bigger than a prescribed tolerance

$$
\Gamma_{\gamma, \mu_{j}}(z)=\frac{1}{\sqrt{2 \pi} \gamma} \exp \left(-\frac{1}{2}\left(\frac{z-\mu_{j}}{\gamma}\right)^{2}\right) \geq \epsilon,
$$

which is given by

$$
-\sqrt{-2 \gamma^{2} \log (\sqrt{2 \pi} \gamma \epsilon)}+\mu_{j} \leq z \leq \sqrt{-2 \gamma^{2} \log (\sqrt{2 \pi} \gamma \epsilon)}+\mu_{j} .
$$

Since we postulate a real solution with $z \in \mathbb{R}$, the term under the square root must be non-negative, i.e.,

$$
\epsilon \leq \frac{1}{\sqrt{2 \pi} \gamma}
$$


That is why we must take care when choosing the tolerance value $\epsilon$ and $\gamma$ when implementing an algorithm as $\gamma$ tends to zero if $\epsilon$ tends to $\infty$ and vice versa.

Now we show that with the restriction on $z$ in (3.2) and therewith the newly found finite limits, namely

$$
\begin{aligned}
& A=-\sqrt{-2 \gamma^{2} \log (\sqrt{2 \pi} \gamma \epsilon)}+\mu_{j}, \\
& B=\sqrt{-2 \gamma^{2} \log (\sqrt{2 \pi} \gamma \epsilon)}+\mu_{j}=-A+2 \mu_{j},
\end{aligned}
$$

we approximate our original integral. If $\epsilon$ tends to zero A tends to $-\infty$ and B to $\infty$, respectively. Every approximation goes hand and hand with its error which occurs through the truncation of the integration interval. Presume our solution is Lipschitz continuous with respect to $x, \tilde{V}(\cdot, \tau) \in \operatorname{Lip}(\mathbb{R})$ with a Lipschitz constant $L$, our approximation error can be estimated by

$$
\begin{aligned}
& \lambda\left(\left|\int_{-\infty}^{\infty}-\int_{-B}^{B}(\tilde{V}(z+x, \tau)-\tilde{V}(x, \tau)) \Gamma_{\gamma, \mu_{j}}(z) \mathrm{d} z\right|\right) \\
= & 2 L \lambda\left(\int_{B}^{\infty}|z| \Gamma_{\gamma, \mu_{j}}(z) \mathrm{d} z\right)<2 \lambda\left(\epsilon L \gamma^{2}+c\right)=\tilde{\epsilon},
\end{aligned}
$$

whereas $c, \epsilon$ and $\gamma$ denote any arbitrary constants satisfying the above conditions.

\subsection{The $\theta$-method}

The $\theta$-method can be used for both PDE and integral term. Since this system of equations leads to a full system matrix Duffy [8] proposed using the Crank-Nicolson method for the PDE term and treating the integral part explicitly in order to get a tridiagonal matrix system. A similar approach was suggested by Cont and Voltchkova [5], where the PDE part is treated implicitly and the integral part explicitly (IMPEX method). While discretizing the scheme, Cont and Voltchkova also differentiated the jump intensity in the finite intensity case, which does not need special treatment, and in the infinite intensity case. The procedure is similar to the truncation of the integral but dealing with small jumps. Cont and Voltchkova [5] replaced the jumps of size smaller $\epsilon$ by a GBM and approximate the Poisson process by an appropriate process possessing jumps of finite intensity. Besides this, a great deal has been done for the convergence properties of this scheme. Usually consistency and stability ensure convergence but we require uniformly bounded derivatives which is simply not the case for swing options. In a thorough manner Cont and Voltchkova showed the monotonicity, consistency and unconditional stability of the proposed scheme by using the so called viscosity method.

Our method is based on the approach of [5], but instead of treating the integral part explicitly we choose to let $\theta_{1}$ and $\theta_{2}$ be any value between zero and 1 . In particular, for $\theta_{2}=1 / 2$ we obtain the Crank-Nicolson scheme for the integral, which is very interesting to investigate as this case delivers a full system matrix which, as far as we say, has never been analysed. Hence, we will focus on solving this issue and will only 
consider the finite density case. For more details how to deal with infinite density see [5]. Now we rewrite our PIDE with its new boundary for the integral to adjust the $\theta$-method

$$
\frac{\partial \tilde{V}}{\partial \tau}=\underbrace{\frac{\sigma^{2}}{2}}_{=c} \frac{\partial^{2} \tilde{V}}{\partial x^{2}}+\underbrace{(r-\delta-\lambda \bar{k})}_{=a} \frac{\partial \tilde{V}}{\partial x}-\underbrace{(r+\lambda)}_{=b} \tilde{V}+\underbrace{\lambda\left(\int_{A}^{B} \tilde{V}(z+x, \tau) \Gamma_{\gamma, \mu_{j}}(z) \mathrm{d} z\right)}_{=\mathcal{I} \tilde{V}} .
$$

To discretize (3.4) we write the convection diffusion part $\mathcal{L}$ of the PIDE explicitly

$$
\mathcal{L}=a \frac{\partial}{\partial x}-b+c \frac{\partial^{2}}{\partial x^{2}}
$$

Now the PIDE (3.4) can be expressed in the compact form

$$
\frac{\partial \tilde{V}}{\partial \tau}=\mathcal{L} \tilde{V}+\mathcal{I} \tilde{V}
$$

Let us define a uniform grid by introducing the grid points $\left(x_{j}, \tau_{i}\right)=(j h, i k)$ with $j \in \mathbb{Z}, i \in \mathbb{N}$, whereas $h$ is the spatial step size and $k$ is the temporal step size

$$
\begin{array}{ll}
x_{j+1}=x_{j}+h, & \forall j \in \mathbb{Z}, \\
\tau_{i+1}=\tau_{i}+k, & \forall i \in\{0, \cdots, I\}, I \in \mathbb{N}, \quad \tau_{0}=0, \quad \tau_{I}=T .
\end{array}
$$

When applying the grid (3.7) and the $\theta$-method on (3.5), we obtain at $x=x_{j}$

$$
\begin{aligned}
\frac{\tilde{V}\left(x, \tau_{i+1}\right)-\tilde{V}\left(x, \tau_{i}\right)}{k}+\mathcal{O}(k)= & \left(1-\theta_{1}\right) \mathcal{L} \tilde{V}\left(x, \tau_{i}\right)+\theta_{1} \mathcal{L} \tilde{V}\left(x, \tau_{i+1}\right) \\
& +\left(1-\theta_{2}\right) \mathcal{I} \tilde{V}\left(x, \tau_{i}\right)+\theta_{2} \mathcal{I} \tilde{V}\left(x, \tau_{i+1}\right)+\mathcal{O}\left(h^{2}\right)
\end{aligned}
$$

In other words

$$
\begin{aligned}
& \frac{\tilde{V}\left(x_{j}, \tau_{i+1}\right)-\tilde{V}\left(x_{j}, \tau_{i}\right)}{k}+\mathcal{O}(k) \\
= & +\theta_{1}\left(c \frac{\tilde{V}\left(x_{j+1}, \tau_{i+1}\right)-2 \tilde{V}\left(x_{j}, \tau_{i+1}\right)+\tilde{V}\left(x_{j-1}, \tau_{i+1}\right)}{h^{2}}+a \frac{\tilde{V}\left(x_{j+1}, \tau_{i+1}\right)-\tilde{V}\left(x_{j-1}, \tau_{i+1}\right)}{2 h}\right. \\
& \left.-b \tilde{V}\left(x_{j}, \tau_{i+1}\right)\right)\left(1-\theta_{1}\right)\left(c \frac{\tilde{V}\left(x_{j+1}, \tau_{i}\right)-2 \tilde{V}\left(x_{j}, \tau_{i}\right)+\tilde{V}\left(x_{j-1}, \tau_{i}\right)}{h^{2}}\right. \\
& \left.+a \frac{\tilde{V}\left(x_{j+1}, \tau_{i}\right)-\tilde{V}\left(x_{j-1}, \tau_{i}\right)}{2 h}-b \tilde{V}\left(x_{j}, \tau_{i}\right)\right)+\mathcal{O}\left(h^{2}\right) .
\end{aligned}
$$

Next we will rewrite the PDE part in matrix form and ignore the integral part for simplicity of the presentation. 


\subsection{Numerical treatment of the PDE part}

A further specification of approximation for the integral part will be discussed in Section 3.3. A pointwise approximation for $\tilde{V}\left(x_{j}, \tau_{i}\right)=\tilde{V}(j h, i k)$ is denoted by $v_{j}^{i}$ where $\tilde{V}(x, \tau)$ is the exact solution for the model problem of (3.4). After sorting all terms according to the time level we approximate this by ignoring terms of $\mathcal{O}(k)$ and $\mathcal{O}\left(h^{2}\right)$

$$
\begin{aligned}
& -v_{j+1}^{i+1}\left(k \theta_{1} \frac{2 c+a h}{2 h^{2}}\right)+v_{j}^{i+1}\left(k \theta_{1} \frac{b h^{2}+2 c}{h^{2}}+1\right)-v_{j-1}^{i+1}\left(k \theta_{1} \frac{2 c-a h}{2 h^{2}}\right) \\
= & v_{j+1}^{i}\left(k\left(1-\theta_{1}\right) \frac{2 c+a h}{2 h^{2}}\right)-v_{j}^{i}\left(k\left(1-\theta_{1}\right) \frac{b h^{2}+2 c}{h^{2}}-1\right)+v_{j-1}^{i}\left(k\left(1-\theta_{1}\right) \frac{2 c-a h}{2 h^{2}}\right) .
\end{aligned}
$$

In order to complete the numerical scheme we need to truncate the infinite mesh at $x_{-J}=-M h$ and $x_{J}=N h$, with $N, M \in \mathbb{N}$ sufficiently large such that the induced errors remain neglectable. At the end, we plot the option price in comparison with the spot price which should be presented by $\exp (x)$. Hence, it is not wise to distribute $x$ equally since 0 would be the middle of the interval $[-M h, \cdots, N h]$ but $\exp (0)$ is quite near the beginning of the interval $[0, \infty)$, i.e., $M$ must be significantly smaller than $N$.

Having applied central differences on the PDE with respect to $x$, these equations are valid for all $j$ except at the boundaries for which a one-sided approximation is needed. Thus, the one-sided differences for (3.6) look like

$$
\frac{\partial \tilde{V}}{\partial x_{J}} \approx \frac{v_{J-1}^{i}-v_{J}^{i}}{h}, \quad \frac{\partial \tilde{V}}{\partial x_{-J}} \approx \frac{v_{-J+1}^{i}-v_{-J}^{i}}{h} .
$$

After a similar calculation the result for the boundaries reads

$$
\begin{aligned}
& v_{-J}^{i+1}\left(-k \theta_{1} \frac{c-b h^{2}-a h}{h^{2}}+1\right)+v_{-J+1}^{i+1}\left(-k \theta_{1} \frac{a h-2 c}{h^{2}}\right)+v_{-J+2}^{i+1}\left(-k \theta_{1} \frac{c}{h^{2}}\right) \\
= & v_{-J}^{i}\left(k\left(1-\theta_{1}\right) \frac{c-b h^{2}-a h}{h^{2}}+1\right)+v_{-J+1}^{i}\left(k\left(1-\theta_{1}\right) \frac{a h-2 c}{h^{2}}\right)+v_{-J+2}^{i}\left(k\left(1-\theta_{1}\right) \frac{c}{h^{2}}\right), \\
& v_{J}^{i+1}\left(-k \theta_{1} \frac{c-b h^{2}-a h}{h^{2}}+1\right)+v_{J-1}^{i+1}\left(-k \theta_{1} \frac{a h-2 c}{h^{2}}\right)+v_{J-2}^{i+1}\left(-k \theta_{1} \frac{c}{h^{2}}\right) \\
= & v_{J}^{i}\left(k\left(1-\theta_{1}\right) \frac{c-b h^{2}-a h}{h^{2}}+1\right)+v_{J-1}^{i}\left(k\left(1-\theta_{1}\right) \frac{a h-2 c}{h^{2}}\right)+v_{J-2}^{i}\left(k\left(1-\theta_{1}\right) \frac{c}{h^{2}}\right) .
\end{aligned}
$$

To write (3.9), (3.11a) and (3.11b) as a linear system, we introduce

$$
A_{1}:=\left(\begin{array}{cccccc}
c-b h^{2}-a h & a h-2 c & c & 0 & \cdots & 0 \\
\frac{2 c-a h}{2} & -b h^{2}-2 c & \frac{2 c+a h}{2} & 0 & \cdots & 0 \\
0 & \frac{2 c-a h}{2} & -b h^{2}-2 c & \frac{2 c+a h}{2} & & 0 \\
\vdots & \ddots & \ddots & \ddots & \ddots & \vdots \\
0 & \ldots & 0 & \frac{2 c-a h}{2} & -b h^{2}-2 c & \frac{2 c+a h}{2} \\
0 & \cdots & 0 & c & a h-2 c & c-b h^{2}-a h
\end{array}\right) .
$$


Now we can write our matrix equation

$$
\left(\mathbf{I}-\alpha \theta_{1} A_{1}\right) v^{i+1}=\left(\mathbf{I}+\alpha\left(1-\theta_{1}\right) A_{1}\right) v^{i},
$$

with the parabolic mesh ratio $\alpha=k / h^{2}, \mathbf{I}$ is the identity matrix and $v^{i}$ is a vector holding the option values at time $\tau_{i}=i k$ for all $x_{j}=j h$ with

$$
v^{i}=\left(v_{-J}^{i}, v_{-J+1}^{i}, \cdots, v_{J-1}^{i}, v_{J}^{i}\right)^{\top}, \quad \forall i \in\{0, \cdots, I\}, I \in \mathbb{N} .
$$

If we use the fact that the boundary conditions of swing options of European type determine both $v_{J}^{i}$ and $v_{-J}^{i}$ with

$$
v_{-J}^{i}=D K e^{-r \tau_{i}} \quad \text { and } \quad v_{J}^{i}=U\left(e^{x_{J}-\delta \tau_{i}}-K e^{-r \tau_{i}}\right), \quad \forall i \in\{1, \cdots, I\}, I \in \mathbb{N},
$$

the corresponding matrix reduces to

$$
A_{2}:=\left(\begin{array}{ccccc}
-b h^{2}-2 c & \frac{2 c+a h}{2} & 0 & \cdots & 0 \\
\frac{2 c-a h}{2} & -b h^{2}-2 c & \frac{2 c+a h}{2} & & \\
0 & \ddots & \ddots & \ddots & \vdots \\
\vdots & & & & 0 \\
0 & \cdots & 0 & \frac{2 c-a h}{2} & -b h^{2}-2 c
\end{array}\right) .
$$

Hence, the matrix equation has a slightly different form

$$
\left(\mathbf{I}-\alpha \theta_{1} A_{3}\right) v^{i+1}=\left(\mathbf{I}+\alpha\left(1-\theta_{1}\right) A_{2}\right) v^{i}+\alpha b^{i},
$$

where

$$
b^{i}=\left(\begin{array}{c}
\left(\frac{2 c-a h}{2}\right) D K\left[\left(1-\theta_{1}\right) e^{-r \tau_{i}}+\theta_{1} e^{-r \tau_{i+1}}\right] \\
0 \\
\vdots \\
0 \\
\left(\frac{2 c+a h}{2}\right) U\left[\left(1-\theta_{1}\right)\left(e^{x_{J}-\delta \tau_{i}}-K e^{-r \tau_{i}}\right)+\theta_{1}\left(e^{x_{J}-\delta \tau_{i+1}}-K e^{-r \tau_{i+1}}\right)\right]
\end{array}\right),
$$

and

$$
v^{i}=\left(v_{-J+1}^{i}, \cdots, v_{J-1}^{i}\right)^{\top}, \quad \forall i \in\{0, \cdots, I\} .
$$

The existence and uniqueness of the solution to (3.12), (3.13) is only guaranteed if the regularity of the matrix

$$
B_{1}:=\mathbf{I}-\alpha \theta_{1} A_{1} \quad \text { and } \quad B_{2}:=\mathbf{I}-\alpha \theta_{1} A_{2},
$$

is warranted. Obviously the regularity holds for $B_{2}$ if it holds for $B_{1}$. 
Definition 3.1. A square matrix $A=\left(A_{i j}\right)$ is called strictly diagonally dominant if

$$
\left|A_{i i}\right|>\sum_{j=1, j \neq i}^{n}\left|A_{i j}\right|, \quad \forall i=1, \cdots, n .
$$

We will show that $B_{1}$ is strictly diagonally dominant that implies the regularity of $B_{1}$. The diagonal elements of $B_{1}$ are $-b h^{2}-2 c$ and $c-b h^{2}-a h$. First, we will derive conditions for $\left|1-\alpha \theta_{1}\left(-b h^{2}-2 c\right)\right|$ being larger than the sum of the magnitudes of the non-diagonal elements in the row,

$$
\begin{aligned}
\left|1-\alpha \theta_{1}\left(-b h^{2}-2 c\right)\right| & =\left|1+\alpha \theta_{1}\left(b h^{2}+2 c\right)+\alpha \theta_{1} \frac{a h}{2}-\alpha \theta_{1} \frac{a h}{2}\right| \\
& =\left|\alpha \theta_{1} \frac{2 c+a h}{2}+\alpha \theta_{1} \frac{2 c-a h}{2}+1+\alpha \theta_{1} b h^{2}\right| .
\end{aligned}
$$

As $2 c$ is positive and constant, we can make $h$ sufficiently small and therewith $a h$ sufficiently small, so that $2 c+a h$ and $2 c-a h$ always remain positive. With this condition, we can write (3.14) in the form

$$
\left|\alpha \theta_{1}\right|\left|\frac{2 c+a h}{2}\right|+\left|\alpha \theta_{1}\right|\left|\frac{2 c-a h}{2}\right|+1+\alpha \theta_{1} b h^{2},
$$

which is obviously larger than

$$
\left|\alpha \theta_{1}\right|\left(\left|\frac{2 c+a h}{2}\right|+\left|\frac{2 c-a h}{2}\right|\right),
$$

and thus, the first inequality is proven and therewith the regularity of $B_{2}$.

To prove the regularity of $B_{1}$, we show this condition for the boundaries:

$$
\left|1-\alpha \theta_{1}\left(c-a h-b h^{2}\right)\right|>\left|\alpha \theta_{1}(2 c+a h)+\alpha \theta_{1} c\right|>\left|\alpha \theta_{1}(2 c-a h)+\alpha \theta_{1} c\right| .
$$

Eq. (3.16) holds if and only if

$$
\left|1-\alpha \theta_{1}\left(c-a h-b h^{2}\right)\right|>\left|\alpha \theta_{1}(2 c+a h)+\alpha \theta_{1} c\right|,
$$

and $a>0$ are true. If $4 c-b h^{2}$ is non-positive, we have to impose the condition $b>$ $4 c / h^{2}$. But for $h$ sufficiently small, $b$ must be very big which is not plausible. However, if we swap the condition with $b<4 c / h^{2}$, more accurately, we postulate that there is a constant $\tilde{r}<1 /\left(\alpha \theta_{1}\right)$ with $4 c=b h^{2}+\tilde{r}$, this inequality would hold in general

$$
1>4 \alpha \theta_{1} c-b h^{2} \alpha \theta_{1}=\alpha \theta_{1}\left(4 c-b h^{2}\right),
$$

and ensures the condition for (3.16). To summarize, we have shown that the matrices $B_{1}, B_{2}$ are strictly diagonally dominant under the following 3 conditions:

1. For any $k$, but $h$ must be sufficiently small to keep $2 c+a h$ and $2 c-a h$ positive.

2. For $4 c=b h^{2}+\tilde{r}$ with $\tilde{r}<1 /\left(\alpha \theta_{1}\right)$.

3. a must be strictly positive.

Thus the matrices $B_{1}$ and $B_{2}$ are invertible. 


\subsection{Numerical treatment of the integral part}

As stated in [6], a classical way of approaching the integral part with numerical integration methods, such as Simpson's rule or Gaussian quadrature, are, regarding computational cost, more expensive than using FFT methods as our integral has a form of a correlation product. The FFT algorithm assumes that the input functions are periodic and it is a matter of fact that neither the option value nor the probability function is periodic. Avoiding this problem by extending the domain on the left and right sides and assuming a linearity in $S$ of the option function $V$, $\mathrm{d}^{\prime}$ Halluin [6] succeed his goal and solved the PIDE in a new region. However, this assumption is only true for portfolios which consist only of call or put options. Numerical solutions for interruptible swing options illustrated in [21, Fig. 4.3] and in [22, Fig. 6.7] support our statement. As soon as we have a combination of call and put options, which is exactly our case, speaking of linearity makes no sense and thus we do not use FFT methods.

The integral of $f$ over an interval $[a, b]$ can be approximated by Simpson's rule as

$$
\int_{a}^{b} f(x) \mathrm{d} x \approx h\left[\sum_{k=1}^{\frac{m}{2}} \frac{4}{3} f\left(x_{2 k-1}\right)+\frac{2}{3} f\left(x_{2 k}\right)+\frac{1}{3}\left(f\left(x_{0}\right)-f\left(x_{m}\right)\right)\right],
$$

where $h=(b-a) / m$ is the distance between adjacent grid points $x_{k}$ and $m \in \mathbb{N}$. Let us recall that when applying Simpson's rule there must be an even number of intervals, i.e., $m=2 n, n \in \mathbb{N}$. We consider the integral term in the PIDE (3.4)

$$
\mathcal{I}=\int_{-\infty}^{\infty} \tilde{V}(x+z, \tau) \Gamma_{\gamma, \mu_{j}}(z) \mathrm{d} z
$$

We could utilize the fact that the probability function $\Gamma_{\gamma, \mu_{j}}(z)$ is similar to the weight function $w(x)=\exp \left(-x^{2}\right)$ of Gauß-Hermite and substitute $z$ to get exactly the same form. Gauß-Hermite is the only method which deals with infinite boundaries. With the change of variables $\tilde{z}=\left(z-\mu_{j}\right) /(\sqrt{2} \gamma)$ the integral (3.20) reads

$$
\mathcal{I}=\frac{1}{\sqrt{\pi}} \int_{-\infty}^{\infty} \tilde{V}(x+\tilde{z}, \tau) \exp \left(-\tilde{z}^{2}\right) \mathrm{d} \tilde{z} .
$$

If $\tilde{V}$ is Lipschitz continuous with respect to $\tau$ the infinite interval can be truncated and we apply the Gauß-Legendre quadrature. Let us recall the integral part of (3.4)

$$
\left(1-\theta_{2}\right) \int_{A}^{B} \tilde{V}\left(x_{j}+z, \tau_{i}\right) \Gamma_{\gamma, \mu_{j}}(z) \mathrm{d} z+\theta_{2} \int_{A}^{B} \tilde{V}\left(x_{j}+z, \tau_{i+1}\right) \Gamma_{\gamma, \mu_{j}}(z) \mathrm{d} z .
$$

We denote

$$
I_{j}^{i} \approx \int_{A}^{B} \tilde{V}\left(x_{j}+z, \tau_{i}\right) \Gamma_{\gamma, \mu_{j}}(z) \mathrm{d} z, \quad I^{i}=\left(I_{-J+1}^{i} I_{-J+1}^{i} I_{-J+2}^{i} \cdots I_{J-2}^{i} I_{J-1}^{i} I_{J-1}^{i}\right)^{\top} .
$$

That means for each $j \in\{-J+1, \cdots, J-1\}$ we have to calculate the integral

$$
\int_{a}^{b} \tilde{V}\left(x_{j}+z, 0\right) \Gamma_{\gamma, \mu_{j}}(z) \mathrm{d} z
$$


with given initial condition $\tilde{V}\left(x_{j}, 0\right)$. Suppose we construct a grid on $[A, B]$, defining a mesh $\tilde{h}=(B-A) / M, M=2 \tilde{N}$, where $\tilde{N}$ is an integer. Thus, $\tilde{h}=B / \tilde{N}$ as $A=-B$. The grid points are $z_{l+1}=z_{l}+\tilde{h}$, for all $l \in\{-\tilde{N}, \cdots, 0, \cdots, \tilde{N}\}$ with the boundaries $z_{-\tilde{N}}=-\tilde{N} \tilde{h}=A$ and $z_{\tilde{N}}=\tilde{N} \tilde{h}=B$. Note that $\tilde{h}$ does not have necessarily to be equal to $h$ but has to be evenly spaced. Without loss of generality we assume that $\tilde{N}$ is even. Then by applying Simpson's rule we obtain the approximation

$$
\begin{aligned}
& \int_{A}^{B} \tilde{V}\left(x_{j}+z, \tau_{i}\right) \Gamma_{\gamma, \mu_{j}}(z) \mathrm{d} z \\
\approx & \sum_{l=1}^{\tilde{N}} \frac{4 \tilde{h}}{3} \tilde{V}\left(x_{j}+z_{-\tilde{N}+(2 l-1)}, \tau_{i}\right) \Gamma_{\gamma, \mu_{j}}\left(z_{-\tilde{N}+(2 l-1)}\right) \\
& +\frac{2 \tilde{h}}{3} \tilde{V}\left(x_{j}+z_{-\tilde{N}+2 l}, \tau_{i}\right) \Gamma_{\gamma, \mu_{j}}\left(z_{-\tilde{N}+2 l}\right) \\
& +\frac{\tilde{h}}{3}\left(\tilde{V}\left(x_{j}+z_{-\tilde{N}}, \tau_{i}\right) \Gamma_{\gamma, \mu_{j}}\left(z_{-\tilde{N}}\right)-\tilde{V}\left(x_{j}+z_{\tilde{N}}, \tau_{i}\right) \Gamma_{\gamma, \mu_{j}}\left(z_{\tilde{N}}\right)\right) \\
\approx & \tilde{h}\left[\sum_{l=1}^{\tilde{N}} \frac{4}{3} v_{j-\tilde{N}+(2 l-1)}^{i} \Gamma_{\gamma, \mu_{j}}\left(z_{-\tilde{N}+(2 l-1)}\right)+\frac{2}{3} v_{j-\tilde{N}+2 l}^{i} \Gamma_{\gamma, \mu_{j}}\left(z_{-\tilde{N}+2 l}\right)\right] \\
& +\frac{\tilde{h}}{3}\left(v_{j-\tilde{N}}^{i} \Gamma_{\gamma, \mu_{j}}\left(z_{-\tilde{N}}\right)-v_{j+\tilde{N}}^{i} \Gamma_{\gamma, \mu_{j}}\left(z_{\tilde{N}}\right)\right) \\
= & I^{i}\left(x_{j}\right)=: I_{j}^{i} .
\end{aligned}
$$

Originally we are interested in calculating $\left\{v_{j}^{i}\right\}_{j \in\{J, \cdots,-J\}}$. However, evaluating the integral requires more values, as values from the interval $[A, B]$ will be added to $x$. We can extend the interval, but this will rise the computational cost enormously. Thus, we will impose the following conditions for all $j \in\{-J, \cdots, J\}$ and $l \in\{-\tilde{N}, \cdots, \tilde{N}\}$ :

$$
\tilde{V}\left(x_{j}+z_{l}\right)= \begin{cases}\tilde{V}\left(x_{J}\right), & \text { if } x_{j}+z_{l} \geq x_{J}, \\ \tilde{V}\left(x_{-J}\right), & \text { if } x_{j}+z_{l} \leq x_{-J}, \\ \tilde{V}\left(x_{j}+z_{l}\right), & \text { otherwise. }\end{cases}
$$

The pointwise approximation of $\tilde{V}\left(x_{j}+z_{l}, \tau_{i}\right)=\tilde{V}(j h+l \tilde{h}, k i)$ will be denoted by $v_{j+l}^{i}$. Our matrix system reads

$$
\begin{aligned}
& \left(\mathbf{I}-\alpha \theta_{1} A_{1}\right) v^{i+1}-k \lambda \theta_{2} I^{i+1}=\left(\mathbf{I}+\alpha\left(1-\theta_{1}\right) A_{1}\right) v^{i}+k \lambda\left(1-\theta_{2}\right) I^{i}, \\
& \underbrace{\left(\mathbf{I}-\alpha \theta_{1} A_{2}\right)}_{A} v^{i+1}-k \lambda \theta_{2} I^{i+1}=\underbrace{\left(\mathbf{I}+\alpha\left(1-\theta_{1}\right) A_{2}\right)}_{q^{i}} v^{i}+\alpha b^{i}+k \lambda\left(1-\theta_{2}\right) I^{i} .
\end{aligned}
$$

Next we state the algorithm to solve (3.22b).

Algorithm 3.1. For each time step $i$ the corresponding option value and integral $v^{i}$ and $I^{i}$ is calculated. Having $v^{i}$ and $I^{i}, v^{i+1}$ is computed, every $v^{i+1}$ is used to recompute the corresponding option value and integral $\tilde{v}^{i}$ and $\tilde{I}^{i}$. If the difference between the old and the new 
integral lies within a tolerance limit tol, we can step forward and increase the time $i$. Otherwise the old integral value will take the new value $\tilde{I}^{i}$ and the whole procedure will be repeated. $i=2$;

$$
\begin{aligned}
& \text { while } i \leq M \\
& \qquad q^{i-1} \leftarrow\left(v^{i-1}, I^{i-1}, I^{i}, b^{i-1}, B\right), \quad \tilde{v}^{i} \leftarrow\left(A, q^{i-1}\right) \quad \tilde{I}^{i} \leftarrow\left(\tilde{v}^{i}, z\right) \\
& \quad \text { if }\left|I^{i}-\tilde{I}^{i}\right| \leq \text { tol then } \\
& \quad v^{i}=\tilde{v}^{i}, \quad i=i+1 \\
& \text { else } \\
& \quad I^{i}=\tilde{I}^{i} \\
& \text { end if } \\
& \text { end while } \\
& \text { option value }=v^{M} .
\end{aligned}
$$

Let us note that by adjusting the parameter set we will obtain either European, Bermudan, American or swing option values.

\subsection{Stability and convergence of the implicit-explicit scheme}

If $\theta_{2}$ is not equal zero, there is no way to solve (3.22b) straightforwardly, because $\tilde{V}\left(x, \tau_{i}\right)$ is needed to calculate $\tilde{V}\left(x, \tau_{i}\right)$. In order to tackle this problem and allow $\theta_{2}$ to be any value between zero and one, we switch the integral's treatment for every time step $i$. In particular, we are going to treat the integral term explicitly. After having the value of $\tilde{V}\left(x, \tau_{i}\right)$ we use this result into a new calculation, in which $\theta_{2} \neq 0$. By comparing the new to the old solution we will decide which treatment is to use in the next step. If the difference lies within a given tolerance limit, we increase the time step and repeat the same procedure. If the difference is larger than allowed we do not increase the time step and repeat the calculation. By treating the PDE part implicitly $\left(\theta_{1}=1\right)$, the above matrix system becomes

$$
\begin{aligned}
& \left(\mathbf{I}-\alpha A_{\xi}\right) v^{i+1}-k \lambda \theta_{2} I^{i+1}=v^{i}+k \lambda\left(1-\theta_{2}\right) I^{i}, \quad \xi=1,2, \\
& \left(\mathbf{I}-\alpha A_{3}\right) v^{i+1}-k \lambda \theta_{2} I^{i+1}=v^{i}+\alpha b^{i}+k \lambda\left(1-\theta_{2}\right) I^{i} .
\end{aligned}
$$

Under some conditions we can prove the stability and convergence of this implicit explicit (IMPEX) scheme

Theorem 3.1 (Stability of scheme (3.23a)). The discretization method (3.23a) is unconditionally stable for any choice of $\theta_{2} \in[0,1]$, provided that the jump intensity $\lambda$ is finite and $\tilde{h} \leq 3 / 4$ and $h$ sufficiently small in order to keep $2 c+$ ah and $2 c-$ ah positive, $a=r-\delta-\lambda \bar{k}$ and $c=\sigma^{2} / 2$.

Proof. Let $v^{i}$ be the vector containing the exact solution components $v_{j}^{i}, \forall j \in\{-J+$ $1, \cdots, J-1\}$. We then simulate the effect of rounding errors by introducing a small error into the initial data

$$
\hat{v}_{j}^{0}=v_{j}^{0}+E_{j}^{0},
$$


where $E_{j}^{0}$ represents a small initial error. Thus with

$$
\hat{v}_{j}^{i}=v_{j}^{i}+E_{j}^{i}
$$

is $E_{j}^{i}$ the error at time step $\mathrm{i}$ and we obtain an equation for the propagation of the perturbation

$$
\begin{aligned}
& E_{j+1}^{i+1}\left(-k \frac{2 c+a h}{2 h^{2}}\right)+E_{j}^{i+1}\left(-k \frac{-b h^{2}-2 c}{h^{2}}+1\right)+E_{j-1}^{i+1}\left(-k \frac{2 c-a h}{2 h^{2}}\right) \\
= & E_{j}^{i}+k \lambda\left(1-\theta_{2}\right) I_{j}^{i+1}+k \lambda \theta_{2} I_{j}^{i},
\end{aligned}
$$

or

$$
\begin{aligned}
& E_{j}^{i+1}\left(k \frac{b h^{2}+2 c}{h^{2}}+1\right) \\
= & E_{j+1}^{i+1}\left(k \frac{2 c+a h}{2 h^{2}}\right)+E_{j-1}^{i+1}\left(k \frac{2 c-a h}{2 h^{2}}\right)+E_{j}^{i}+k \lambda\left(1-\theta_{2}\right) I_{j}^{i+1}+k \lambda \theta_{2} I_{j}^{i} .
\end{aligned}
$$

We use the maximum norm

$$
\|E\|_{\infty, i}:=\max _{j}\left|E_{j}\right|^{i},
$$

and find

$$
\begin{aligned}
\left|I_{j}^{i}\right|= & \mid \sum_{l=1}^{\tilde{N}} \frac{4 \tilde{h}}{3} E_{j-\tilde{N}+(2 l-1)}^{i} \Gamma_{\gamma}\left(z_{-\tilde{N}+(2 l-1)}\right)+\frac{2 \tilde{h}}{3} E_{j-\tilde{N}+2 l}^{i} \Gamma_{\gamma}\left(z_{-\tilde{N}+2 l}\right) \\
& +\frac{\tilde{h}}{3}\left(E_{j-\tilde{N}}^{i} \Gamma_{\gamma}\left(z_{-\tilde{N}}\right)-E_{j+\tilde{N}}^{i} \Gamma_{\gamma}\left(z_{\tilde{N}}\right)\right) \mid \\
\leq & \frac{4 \tilde{h}}{3}\|E\|_{\infty}^{i}\left|\sum_{l=1}^{\tilde{N}}\left(\Gamma_{\gamma}\left(z_{-\tilde{N}+(2 l-1)}\right)+\left(\Gamma_{\gamma}\left(z_{-\tilde{N}+2 l}\right)\right)+\Gamma_{\gamma}\left(z_{-\tilde{N}}\right)-\Gamma_{\gamma}\left(z_{\tilde{N}}\right)\right)\right| \\
= & \frac{4 \tilde{h}}{3}\|E\|_{\infty}^{i}\left|\sum_{l=-\tilde{N}}^{\tilde{N}-1} \Gamma_{\gamma}\left(z_{l}\right)\right| \leq\|E\|_{\infty, i},
\end{aligned}
$$

as $\Gamma_{\gamma}$ is a density function and $4 \tilde{h} / 3 \leq 1$. With this result and the above-imposed conditions, the magnitude of (3.24) reads

$$
\begin{aligned}
& \left|E_{j}^{i+1}\left(k \frac{b h^{2}+2 c}{h^{2}}+1\right)\right| \\
= & \left|E_{j+1}^{i+1} k\left(\frac{2 c+a h}{2 h^{2}}\right)+E_{j-1}^{i+1} k\left(\frac{2 c-a h}{2 h^{2}}\right)+E_{j}^{i}+k \lambda\left(1-\theta_{2}\right) I_{j}^{i+1}+k \lambda \theta_{2} I_{j}^{i}\right|,
\end{aligned}
$$

and summarizing gives

$$
\begin{aligned}
& {\left[k \frac{b h^{2}+2 c}{h^{2}}+1\right]\left|E_{j}^{i+1}\right| } \\
\leq & k\left(\frac{2 c}{h^{2}}\right)\|E\|_{\infty, i+1}+\|E\|_{\infty, i}+k \lambda\left(1-\theta_{2}\right)\|E\|_{\infty, i+1}+k \lambda \theta_{2}\|E\|_{\infty, i},
\end{aligned}
$$


i.e.,

$$
\left[k \frac{b h^{2}+2 c}{h^{2}}+1\right]\left|E_{j}^{i+1}\right| \leq k\left(\frac{2 c}{h^{2}}+\lambda\left(1-\theta_{2}\right)\right)\|E\|_{\infty, i+1}+\left(1+k \lambda \theta_{2}\right)\|E\|_{\infty, i} .
$$

Let $m$ be an arbitrary but fixed index element with the property

$$
\max _{j \in\{-J+1, \cdots, J-1\}}\left|E_{j}^{i+1}\right|=\left|E_{m}^{i+1}\right| .
$$

Since (3.27) holds for all $j \in\{-J+1, \cdots, J-1\}$, it is particularly valid for $j \equiv m$. Replacing $j$ by $m$ we obtain

$$
\left[k \frac{b h^{2}+2 c}{h^{2}}+1\right]\|E\|_{\infty, i+1} \leq k\left(\frac{2 c}{h^{2}}+\lambda\left(1-\theta_{2}\right)\right)\|E\|_{\infty, i+1}+\left(1+k \lambda \theta_{2}\right)\|E\|_{\infty, i},
$$

and rearranging gives

$$
[k(r+\lambda)+1]\|E\|_{\infty, i+1} \leq k\left(\lambda\left(1-\theta_{2}\right)\right)\|E\|_{\infty, i+1}+\left(1+k \lambda \theta_{2}\right)\|E\|_{\infty, i}
$$

or

$$
\left[1+k\left(r+\lambda \theta_{2}\right)\right]\|E\|_{\infty, i+1} \leq\left(1+k \lambda \theta_{2}\right)\|E\|_{\infty, i}
$$

and therefore,

$$
\|E\|_{\infty, i+1} \leq \frac{\left(1+k \lambda \theta_{2}\right)}{\left[1+k\left(r+\lambda \theta_{2}\right)\right]}\|E\|_{\infty, i} \leq\|E\|_{\infty, i} .
$$

The solutions of (3.23a) are uniformly bounded (with respect to $j$ ) as $i$ tends to $\infty$ for all starting values $\left\{v_{j}^{0}\right\}$, i.e., (3.23a) is stable.

Note that since $x_{J}^{i}$ and $x_{-J}^{i}$ are arbitrary in (3.23a), (3.23b) is also unconditionally stable. The convergence of the scheme (3.23a) can be shown analogously.

\section{Numerical results}

First of all, a simple one factor spot price model is utilized for the valuation of swing call, swing put and interruptible swing options with and without jumps. Furthermore, the impact of parameter values on swing options prices are examined. Finally, a comparison of our calculated swing options values of Bermudan type to swing options values in [22] is performed.

\subsection{Swing options without jumps}

To gain some insight into the numerical valuation of swing contracts we start with the simplest examples, namely swing call and swing put options under the Black-Scholes model, i.e., swing options without jumps. For a start we will analyse the dependence between swing options and the number of exercise rights. The parameter set is taken from Wilhelm [22]. 
In her thesis [22] Wilhelm presented numerical results for swing put options in a Black-Scholes market as well as swing call and swing interruptible options in a electricity market. In the latter market a mean reverting, seasonal spot price model has been considered whereas a simple spot price model with constant interest rate and volatility has been incorporated in the Black-Scholes market.

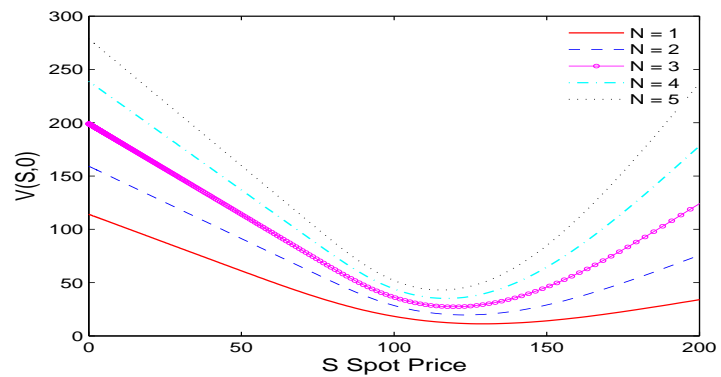

\begin{tabular}{||l|c||}
\hline Parameter & Value \\
\hline expiry date $T$ & 1 \\
volatility $\sigma$ & 0.3 \\
interest rate $r$ & 0.05 \\
strike price $K$ & 100 \\
exercise rights $N$ & $1-5$ \\
refraction period & 0.1 \\
\hline
\end{tabular}

Figure 3: Interruptible swing options with the parameter set of Wilhelm [22], whereas in this case different strike prices are considered, namely $K_{p}=180$ and $K_{c}=120$.

The numerical approximation of swing option prices for up to five exercise rights are presented in the Fig. 3. At a first glance our numerical results for $S=0$ to $S=100$ look similar to the numerical results of [22, Fig. 6.1] for swing put options in a BlackScholes market. However, the maximum values of our swing put options of Bermudan type are smaller than the maximum values of swing put options of American type. The highest pay-off value for a swing put option of Bermudan type lies at $330 €$ compared to $500 €$, which remarks the highest pay-off value for a swing put option of American type. This can be explained as follows. Although the parameter set is the same, but since the refraction period is 0.1 and the expiry date 1 , the resulting exercise opportunities for swing options of Bermudan type is maximum 10, whereas the exercise opportunities for swing options of American type is at least 10. Besides, it does make sense that swing options of American type are equal to or more expensive than swing options of Bermudan type as American options are equal to or more expensive than Bermudan options.

Looking at [22, Fig. 6.4] illustrating swing call options prices, we cannot detect any similarities to our numerical results for $S \geq K_{c}$. The fact that Wilhelm calculated swing call options in an electricity market, not in a Black-Scholes market, explains why her swing options are more expensive than ours. In other words, swing options in an

Table 1: Data used in the interruptible swing options example.

\begin{tabular}{||l|c||}
\hline Parameter & Value \\
\hline expiry date $T$ & 1 \\
volatility $\sigma$ & 0.3 \\
interest rate $r$ & 0.05 \\
strike price $K$ & 10 \\
dividend yields $\delta$ & 0 \\
\hline
\end{tabular}


Table 2: Swing parameters.

\begin{tabular}{||l|c|l|c||}
\hline Parameter & Value & Parameter & Value \\
\hline expiry date $T$ & 1 & exercise opportunities $n$ & 50 \\
volatility $\sigma$ & 0.35 & down-volume $\delta_{\downarrow}$ & 1 \\
interest rate $r$ & 0.1 & up-volume $\delta_{\uparrow}$ & 1 \\
strike price $K$ & 15 & $\theta_{1}$ & 0.5 \\
exercise rights $N$ & 12 & Number of time steps $M$ & 160 \\
\hline
\end{tabular}

electricity market are more expensive than swing options in a Black-Scholes market. The same holds for interruptible swing options.

For another parameter set presented in Table 1 we investigated interruptible swing option prices depending on the number of rights while altering the number of up- and downswings. However, the swing option price per right decreases with increasing number of rights and tends to an equilibrium. This reflects the fact that multiple swing rights cannot be exercised at the same time. The optimal exercise date for the first right, which returns the most profit, cannot be used for the other swings. Fig. 4(a) illustrates this fact.

Let us recall Example 2.1 introduced in Section 2. In the following we will present several numerical solutions for the swing option in this example, depending on the spot prices and time parameters. Assuming the current spot price is $20 €$, the corresponding penalty function is given by

$$
\rho(x)= \begin{cases}15, & \text { if } x<-2 \\ 0, & \text { if }-2 \leq x \leq 2, \\ 20(x-2), & \text { if } x>2\end{cases}
$$

where $x$ denotes the total amount of consumption. The notation is chosen to be conform with the definition of the swing options in Section 2.

Fig. 4 shows the structure of the price of swing options by using the penalty function $\rho$ from Section 4.1, the parameters from Table 2 without jumps, computed with the Crank-Nicolson scheme.

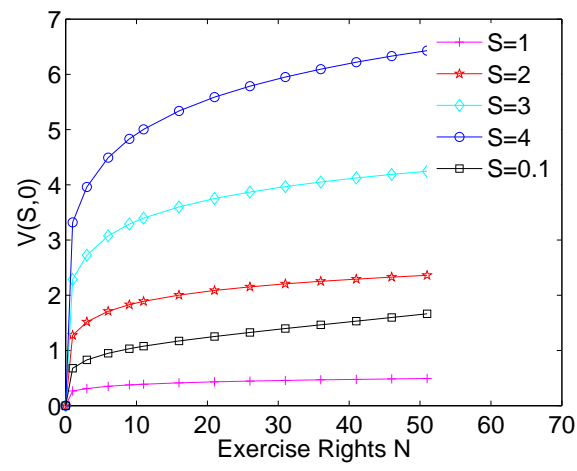

(a) $N=10$

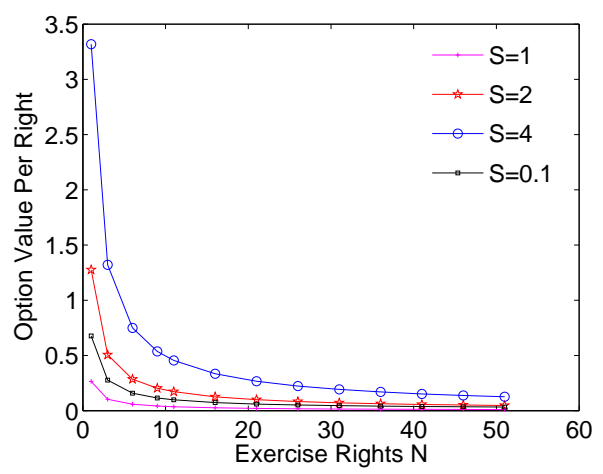

(b) $U=3, D=1$

Figure 4: Comparing a swing option per right at one point price $S$ with increased exercise rights. Parameter set: strike price $K=1$, dividend $\delta=0$, volatility $\sigma=0.2$, interest rate $r=0.25$, expiration date $T=1$ and number of upswings and downswings $U=1, D=1$. 
The effect of the penalty function is illustrated through the upper bounds of the option values, $130 €$ in the left figure compared to $110 €$ in the right figure which leads to the obvious conclusion that a penalty, aside from exceptional cases, decreases the values.

Going on with Example 2.1 we examine the effect of a dividend yield on swing option values for different values of allowed up- and downswings. The swing option prices at time $t=0$ for up to $20 \%$ dividend yield are presented in Fig. 6. Similar to plain Vanilla options, the value of swing call options clearly decrease by higher percentage of the dividend yield, whereas the value of swing put options increase by a higher percentage of dividend yield. Although the number of exercise rights and opportunities in three cases is the same, the effect of different number of upswings and downswings allowed is obvious. The upper boundary in values of the interruptible swing options lie at approximately $50 €$ on the left and $230 €$ on the right, whereas the swing put options reach its maximum at $80 €$ and the swing call options at $300 €$. At this point the reader could ask why the upper boundary in values of the interruptible swing options is not at $80 €$ and $300 €$. One possible explanation is that when $K-S_{t_{i}}>$ $0, t_{i}$ denotes one exercise opportunity, the owner of the swing put option would rather exercise his rights compared to a interruptible swing option holder because the latter still has the opportunity in the future $t_{j}$ to gain higher profit when $S_{t_{j}}-K \geq K-S_{t_{i}}$ happens. In other words, the 12 rights have to be distributed among 12 upswings and 12 downswings whereas in case of call or put swing option the 12 rights can be concentrated within the 12 upswings or downswings.

Now, the fair prices of swing call options at time $t=0$ for different expiry dates will be investigated. Generally, the more time remains before an option expires, the higher the option price. This is not surprising when we recall that option writers demand higher prices when they expect their risk to be greater. This impression is confirmed in Fig. 7(a), where option prices increase if the time to expiration increases. In each graph we change the expiry parameter by $100 \%$ up, starting with $T=0.1$. However, looking at Fig. 7(b), option prices seem to increase linearly with the initial spot price. One observes that the dependence of the options value on the expiry date gets weaker as the expiry is further away.

\subsection{Swing options with jumps}

In this subsection we consider a swing option under a jump diffusion process with a duration of 3 months and 30 exercise rights. As stated in Section 2 we choose the classical Merton's model to describe the distribution of the random variable in the SDE (2.8). First we start to evaluate the swing option for which market parameters are listed in Table 3. Numerical solution of this swing option is plotted in the Figures below as red curves.

Based on the parameter set in Table 3 we plot swing option values by altering each parameter by $50 \%$ up and down. In doing so, we vary swing options in swing call options, swing put options and interruptible swing options and give numerical 

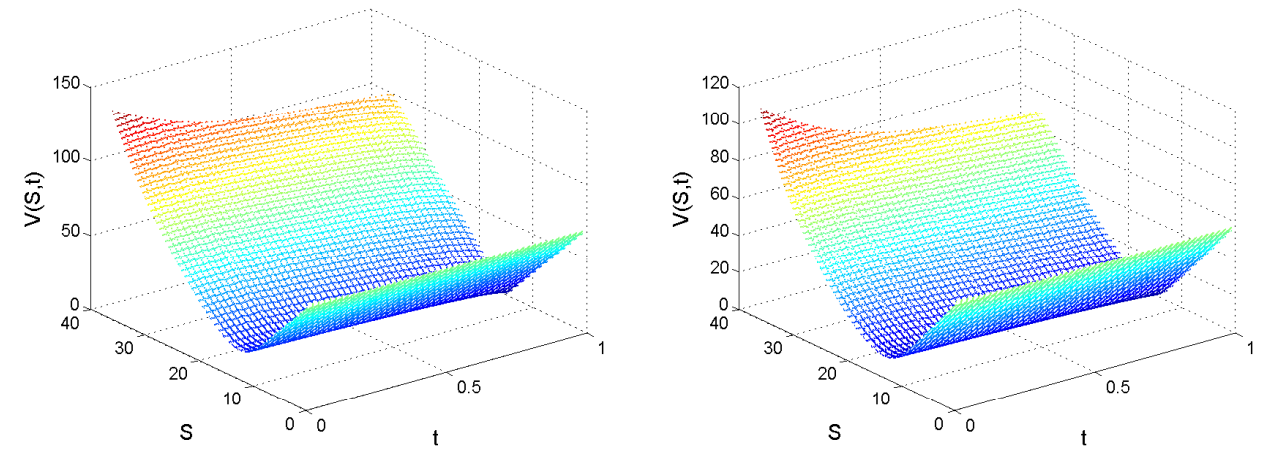

Figure 5: Value of swing options with 12 upswing rights and 12 downswing rights. On the left no penalty has been applied whereas the penalty function (4.1) has been incorporated in the right plot.

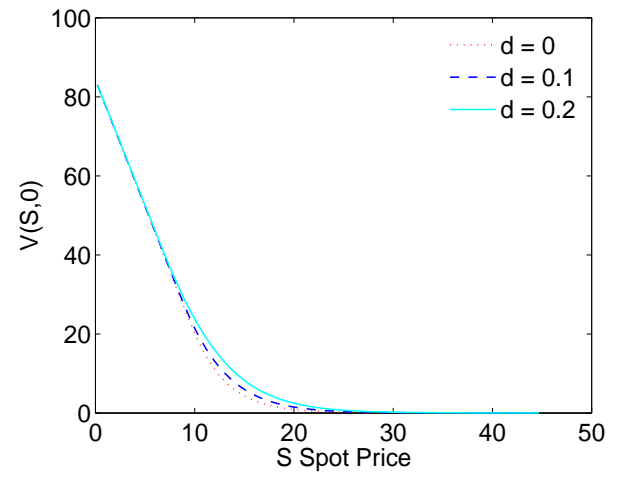

(a) Swing put option $(U, D)=(0,12)$ with various dividend yields $d$.

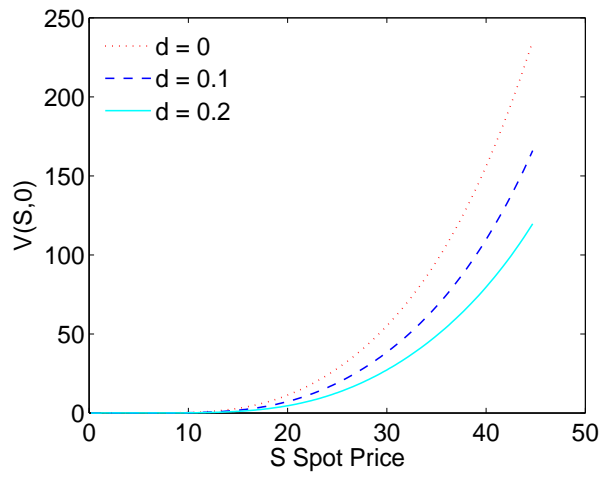

(b) Swing call option $(U, D)=(12,0)$ with various dividend yields $d$.

Figure 6: The influence of a dividend yield on swing options with parameters of Table 2.

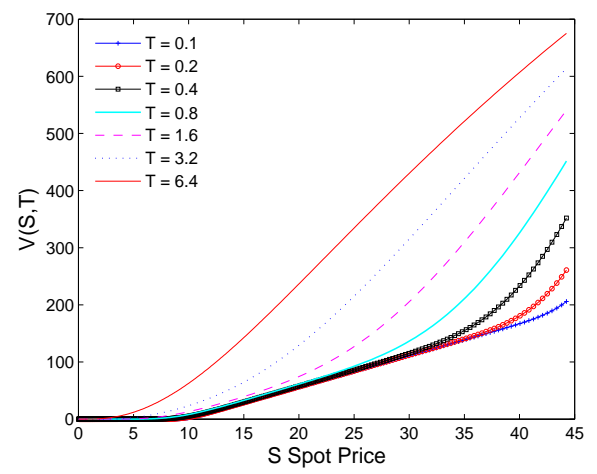

(a) Swing call options for up to seven different expiry dates $T=0.1,0.2, \cdots, 6.4$.

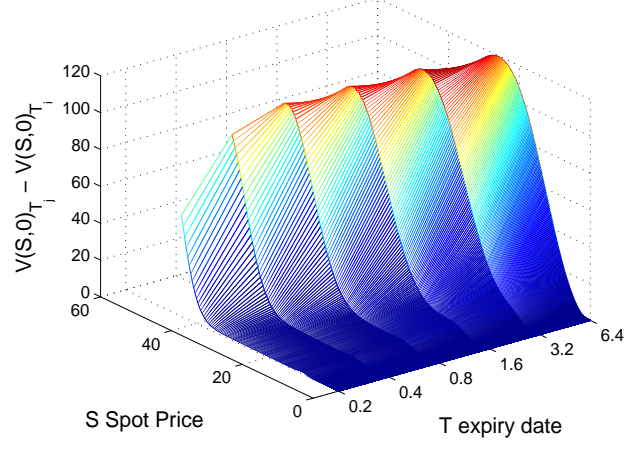

(b) The difference of swing call options of different expiry dates, whereas $T_{j}=2 T_{i}$ and $T_{i}, T_{j} \in\{0.1,0.2, \cdots, 3.2,6.4\}$.

Figure 7: Impact of expiry dates on swing call options. 
Table 3: Data used in the swing option example with jumps.

\begin{tabular}{||l|c|l|c||}
\hline Parameter & Value & Parameter & Value \\
\hline expiry date $T$ & 0.25 & dividend yields $\delta$ & 0 \\
volatility $\sigma$ & 0.35 & exercise opportunities $n$ & 30 \\
interest rate $r$ & 0.1 & jump intensity $\lambda$ & 3 \\
strike price $K$ & 15 & density function of Merton's model & $\gamma=2, \mu_{j}=-3$ \\
\hline
\end{tabular}

solutions for both density functions. Comparing the Fig. 8 of the first case, where the mean of the density function is $\mu_{j}=0.3$, to the Fig. 9 of the second case we make three interesting observations.

First, curves of the first case all have a big "tummy" whereas curves of the second case have tails. More precise, the swing option values of the first case make bigger changes in the middle area of the spot price than at the boundaries, whereas these changes in the swing option prices of the second case shift to the right boundary and are smaller. Recall that the only difference between the two density functions is the mean $\mu_{j}$. Hence, this difference delivers the plausible reason for the shift in the changes of swing option values because the maximum of the density function lies at $\mu_{j}$. Second, the biggest change is caused by a change in the standard deviation $\gamma$ of the corresponding density function. In others words, the volatility of the random variable

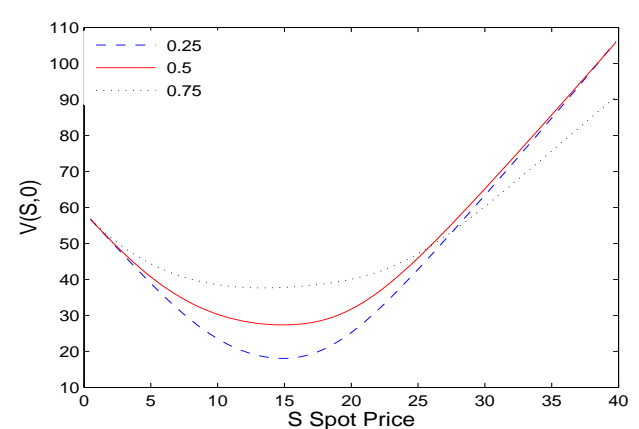

(a) $\gamma$

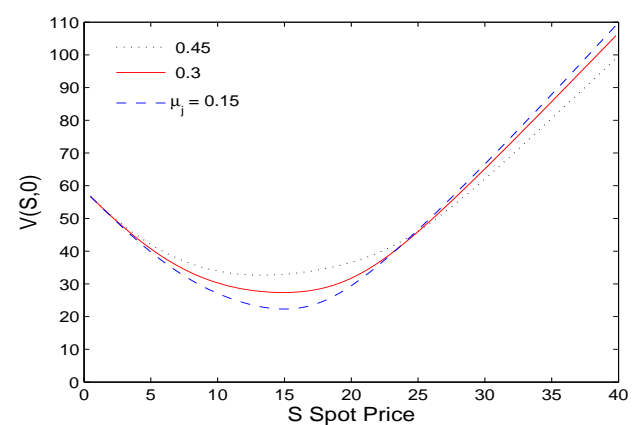

(c) $\mu_{j}$

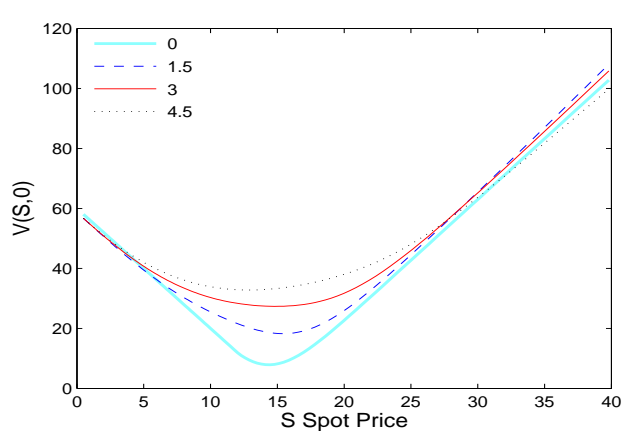

(b) $\lambda$

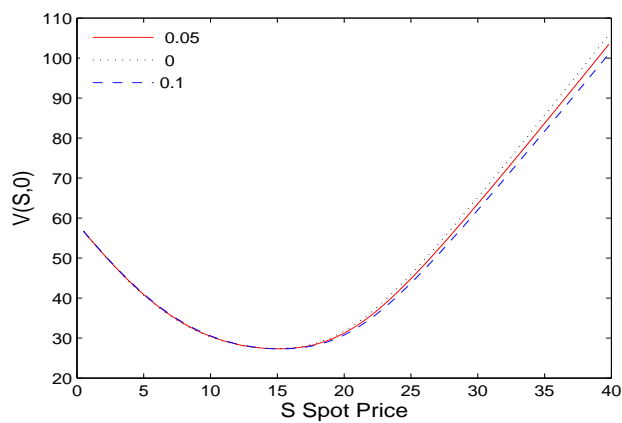

(d) $\delta$

Figure 8: Value of interruptible swing options with 10 upswing rights and 10 downswing rights and $\mu_{j}=0.3$, model parameters as in Table 3. 


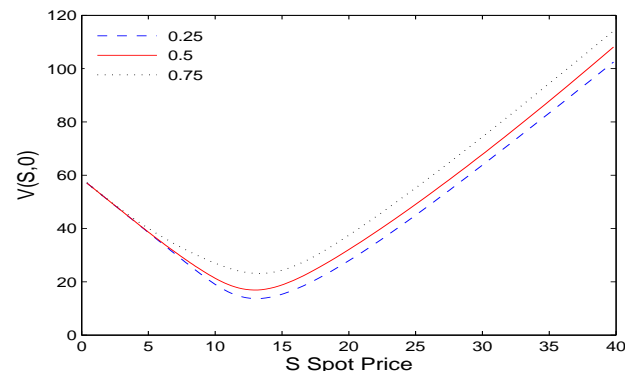

(a) $\gamma$

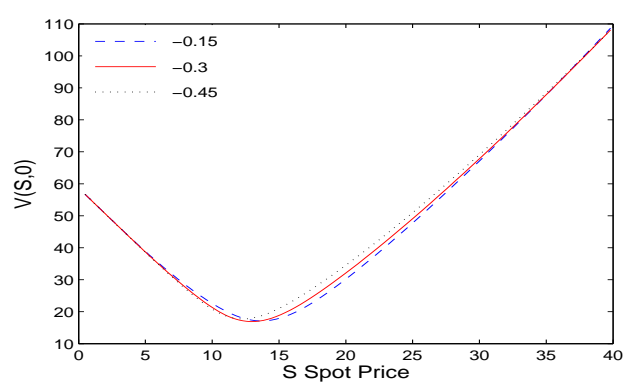

(c) $\mu_{j}$

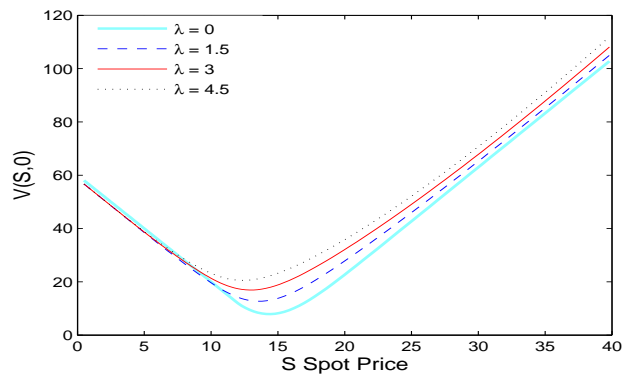

(b) $\lambda$

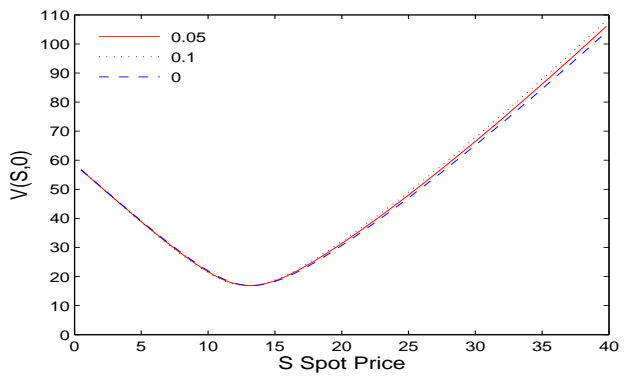

(d) $\delta$

Figure 9: Value of interruptible swing options with 10 upswing rights and 10 downswing rights and $\mu_{j}=-0.3$, model parameters as in Table 3.

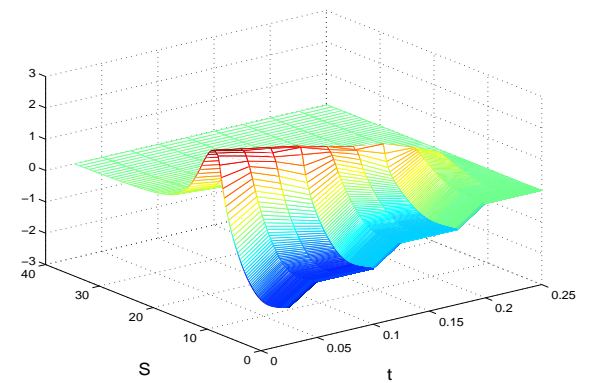

(a) $V(S, t)_{\lambda 2}-V(S, t)_{\lambda 1}$.

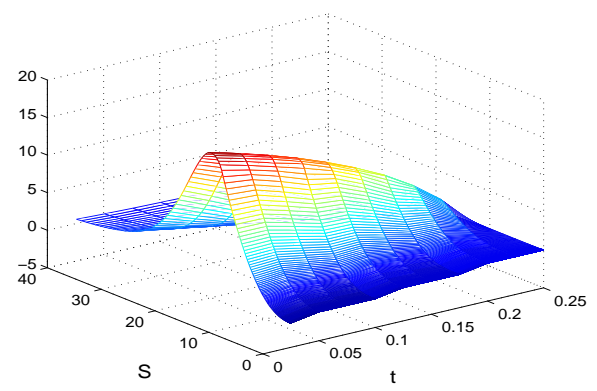

(c) $V(S, t)_{\lambda 4}-V(S, t)_{\lambda 1}$.

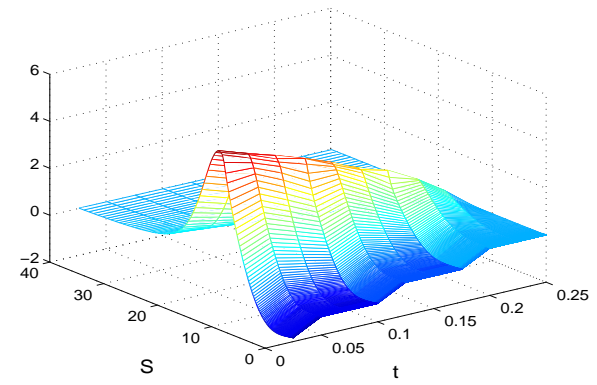

(b) $V(S, t)_{\lambda 3}-V(S, t)_{\lambda 1}$.

Figure 10: Influence of jumps on swing put options with ten rights, whereas $\lambda_{1}=0, \lambda_{2}=0.5, \lambda_{3}=1$ and $\lambda_{4}=4$. It is interesting to see that, around the strike price $K$, the higher the jump intensity the higher the swing option values. However, when the spot price $S$ tends to 0 swing option values decrease. The model parameters are taken from Table 3 . 


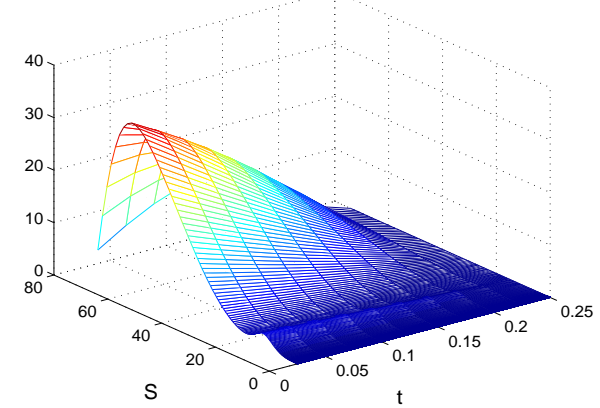

(a) $V(S, t)_{\lambda 2}-V(S, t)_{\lambda 1}$.

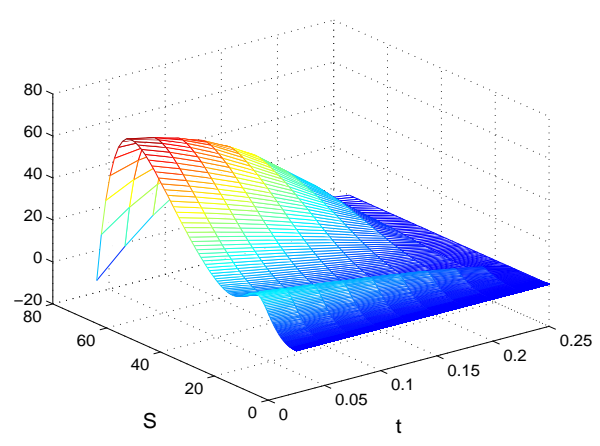

(c) $V(S, t)_{\lambda 4}-V(S, t)_{\lambda 1}$.

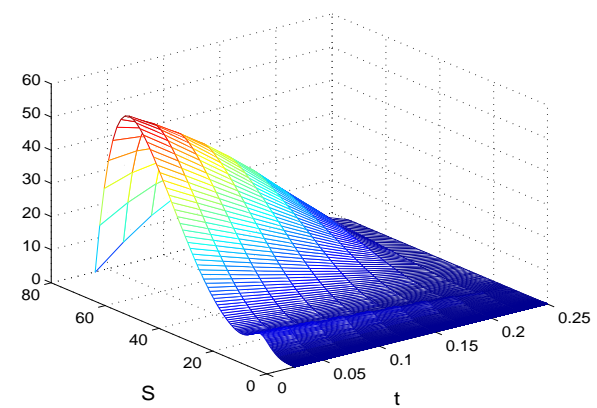

(b) $V(S, t)_{\lambda 3}-V(S, t)_{\lambda 1}$.

Figure 11: Influence of jumps on swing call options with ten rights, whereas $\lambda_{1}=0, \lambda_{2}=0.5, \lambda_{3}=1$ and $\lambda_{4}=1$. For swing call options we observe the same effects as for swing put options. The only difference is that when the spot price $S$ tends to infinity, after reaching their maximum, swing option values decrease. The model parameters are taken from $\mathrm{Ta}$ ble 3 .

in our SDE has a bigger impact on the swing option values than the jump intensity.

Finally, a remarkable observation can be made about the relation between the jump intensity and the swing option values. Similar to the relation between the volatility and the swing option values one might think the higher the jump intensity the higher the option price. In general this is not true, interestingly. For moderate jump values swing options are more expensive, but if the jump parameter $\lambda$ is too high, swing option values decrease near the boundaries. Numerical values in Table 3 underline this effect. In particular, for $\lambda=4$ some swing options values in the sixth column are larger than the swing option values for $\lambda=2$.

A possible explanation is the following: if the jump intensity is too high, the possibility that the spot price jumps into the wrong direction is higher than usual. For a swing call option holder the spot price might decrease significantly and for a swing put option holder the spot price might increase.

Table 4 shows the values of swing call options based on the model parameters of Table 3. The numbers in the first column are the spot prices, the second column gives the numerical values of the swing call option without jump, the numbers in the third, fourth, fifth and sixth column are the numerical values if the same swing option with different jump intensity. The numbers in columns 6 to 8 are percentage values which show the growth rate of a swing call option under different jump intensities. As expected, by doubling the jump intensity $\lambda$ from 0.5 to 1 or from 1 to 2 the growth 
Table 4: The impact of jumps on swing call option prices. Parameter set is taken from Table $3, \mu_{j}=0.3$.

\begin{tabular}{||c|c|c|c|c|c|c|c|c||}
\hline $\mathrm{S}$ & \multicolumn{7}{|c|}{ Option Value } & \multicolumn{3}{c||}{ Growth in Percent } \\
\hline & $\lambda=0$ & $\lambda=0.5$ & $\lambda=1$ & $\lambda=2$ & $\lambda=4$ & $0-0.5$ & $0-1$ & $0-2$ \\
\hline 7.943 & 0.0006 & 1.007 & 1.926 & 3.591 & 6.388 & & & \\
10.000 & 0.053 & 2.016 & 3.740 & 6.616 & 10.762 & & & \\
11.749 & 0.608 & 3.316 & 5.787 & 9.899 & 15.343 & 444.9 & 850.9 & 1526.6 \\
13.803 & 3.577 & 6.362 & 9.258 & 14.579 & 21.630 & 77.8 & 158.8 & 307.5 \\
15.848 & 10.274 & 12.378 & 14.933 & 20.451 & 28.745 & 20.4 & 45.3 & 99.0 \\
17.782 & 19.258 & 20.812 & 22.826 & 27.661 & 36.196 & 8.0 & 18.5 & 43.6 \\
19.952 & 30.756 & 32.242 & 33.978 & 37.947 & 45.493 & 4.8 & 10.4 & 23.3 \\
21.877 & 41.367 & 43.203 & 45.052 & 48.671 & 54.731 & 4.4 & 8.9 & 17.6 \\
23.988 & 53.108 & 55.635 & 57.881 & 61.586 & 66.001 & 4.7 & 8.9 & 15.9 \\
25.704 & 62.670 & 65.942 & 68.654 & 72.680 & 76.012 & 5.2 & 9.5 & 15.9 \\
27.542 & 72.920 & 77.154 & 80.480 & 84.997 & 87.379 & 5.8 & 10.3 & 16.5 \\
29.512 & 83.904 & 89.347 & 93.447 & 98.632 & 100.196 & 6.4 & 11.3 & 17.5 \\
31.622 & 95.674 & 102.596 & 107.637 & 113.621 & 114.361 & 7.2 & 12.5 & 18.7 \\
33.884 & 108.286 & 116.996 & 123.186 & 130.168 & 130.058 & 8.0 & 13.7 & 20.2 \\
35.481 & 117.191 & 127.267 & 134.333 & 142.070 & 141.375 & 8.5 & 14.6 & 21.2 \\
37.153 & 126.516 & 138.109 & 146.150 & 154.709 & 153.311 & 9.1 & 15.5 & 22.2 \\
39.810 & 141.333 & 155.492 & 165.215 & 175.236 & 172.698 & 10.0 & 16.8 & 23.9 \\
41.686 & 151.797 & 167.834 & 178.804 & 189.927 & 186.585 & 10.5 & 17.7 & 25.1 \\
43.651 & 162.755 & 180.800 & 193.118 & 205.443 & 201.168 & 11.0 & 18.6 & 26.2 \\
45.708 & 174.234 & 194.397 & 208.162 & 221.835 & 216.687 & 11.5 & 19.4 & 27.3 \\
\hline
\end{tabular}

Table 5: Summarizing our results about the impact of some model parameters on swing options, this table shows the effect on the value of swing call and put options of an increase of each of the six parameters. Although jump parameters $\lambda, \gamma$ and $\mu_{j}$ have a great effect on swing option prices, a generalization about their relationship to swing option prices is not possible.

\begin{tabular}{||l|c|c||}
\hline Parameter & Swing Call & Swing Put \\
\hline Spot Price & $\Uparrow$ & $\Downarrow$ \\
Strike Price & $\Downarrow$ & $\Uparrow$ \\
Time to expiry & $\Uparrow$ & $\Uparrow$ \\
Dividend yield & $\Uparrow$ & $\Downarrow$ \\
Number of exercise rights & $\Uparrow$ & $\Uparrow$ \\
Volume restriction & $\Downarrow$ & $\Downarrow$ \\
\hline
\end{tabular}

of the option values in percent makes a similar effect, $4.8 \%$ to $10.4 \%$ or from $10.4 \%$ to $23.3 \%$, depending on the exercise opportunities and moneyness of the options.

\section{References}

[1] A. Almendral And C. W. OOsterlee, Numerical valuation of options with jumps in the underlying, Appl. Numer. Math., 53 (2005), pp. 1-18.

[2] J. ANKUDINOVA AND M. EHRHARDT, On the numerical solution of nonlinear Black-Scholes equations, Comput. Math. Appl., 56 (2008), pp. 799-812.

[3] M. BRIANI, C. L. CHIOMA AND R. NATALINI, Convergence of numerical schemes for viscosity solutions to integro-differential degenerate parabolic problems arising in financial theory, 
Numer. Math., 98 (2004), pp. 607-646.

[4] M. BRIANI, R. NATALINI AND G. RUSSO, Implicit-explicit numerical schemes for jumpdiffusion processes, Calcolo., 44 (2007), pp. 33-57.

[5] R. CONT AND E. VOLTCHKOVA, A finite difference scheme for option pricing in jump diffusion and exponential Lévy models, SIAM J. Numer. Anal., 43 (2005), pp. 1596-1626.

[6] Y. D'HALLUin, Numerical Methods for Real Options in Telecommunications, Ph.D. Thesis, University of Waterloo, 2004.

[7] U. DÖRR, Valuation of Swing Options and Examination of Exercise Strategy by Monte Carlo Techniques, Master Thesis, University of Oxford, 2003.

[8] D. J. DufFY, Numerical Analysis of Jump Diffusion Models: A Partial Differential Equation Approach, Working Paper, 2004.

[9] M. EHRHARDT AND R. MICKENS, A fast, stable and accurate numerical method for the BlackScholes equation of American options, Int. J. Theor. Appl. Fina., 11 (2008), pp. 471-501.

[10] G. HAARBRÜCKER AND D. KUHN, Valuation of electricity swing options by multistage stochastic programming, Automatica., 45 (2009), pp. 889-899.

[11] B. HAMbly, S. HowISON AND T. Kluge, Modelling spikes and pricing swing options in electricity markets, Quant. Fina., 9 (2009), pp. 937-949.

[12] A. IBANEZ, Valuation by simulation of contingent claims with multiple early exercise opportunities, Math. Fina., 14 (2004), pp. 223-248.

[13] P. Jaillet, E. RONN AND S. TOMPAidis, Valuation of Commodity-Based Swing Options, Working Paper, 2004.

[14] S. G. KERN, Die Stochastische Modellierung des EEX-Spotmarktes und die Bewertung von Swing-Optionen, Ph.D. Thesis, Technische Universität Graz, 2006.

[15] M. KJAER, Pricing of swing options in a mean reverting model with jumps, Appl. Math. Fina., 155 (2009), pp. 479-502.

[16] T. KLuge, Pricing Swing Options and Other Electricity Derivatives, Ph.D. Thesis, University of Oxford, 2006.

[17] S. G. KOU, A jump diffusion model for option pricing, Manage. Sci., 48 (2002), pp. 1086-1101.

[18] A. MAYO, Methods for the rapid solution of the pricing PIDEs in exponential and Merton models, J. Comput. Appl. Math., 222 (2008), pp. 128-143.

[19] R. C. MERTON, Option pricing when the underlying stocks are discontinuous, J. Fina. Econ., 5 (1976), pp. 125-144.

[20] J. TOIVANEN, Numerical valuation of european and american options under Kou's jumpdiffusion models, SIAM J. Sci. Comput., 30 (2008), pp. 1949-1970.

[21] T. Wegner, Swing Options and Seasonality of Power Prices, Master thesis, University of Oxford, 2002.

[22] M. Wilhelm, Modeling, Pricing and Risk Management of Power Derivatives, Ph.D. Thesis, ETH Zürich, 2007. 